\title{
Circadian Polymorphisms in Night Owls, in Bipolars, and in Non-24-Hour Sleep Cycles
}

\author{
Daniel F. Kripke ${ }^{1,2} \bowtie$, Walter T. Klimecki ${ }^{3}$, Caroline M. Nievergelt ${ }^{1}$, Katharine M. Rex ${ }^{1}$, Sarah S. Murray ${ }^{4}$, \\ Tatyana Shekhtman ${ }^{1,5}$, Gregory J. Tranah ${ }^{6}$, Richard T. Loving ${ }^{7}$, Heon-Jeong Lee ${ }^{8}$, Min Kyu Rhee ${ }^{9}$, \\ Farhad F. Shadan ${ }^{2}$, J. Steven Poceta ${ }^{2}$, Shazia M. Jamil2 ${ }^{2}$ Lawrence E. Kline ${ }^{2}$, and John R. Kelsoe ${ }^{1,5}$ \\ 'Department of Psychiatry, University of California, San Diego, CA, USA \\ ${ }^{2}$ Viterbi Family Sleep Center, Scripps Clinic, La Jolla, CA, USA \\ ${ }^{3}$ Department of Pharmacology and Toxicology, University of Arizona, Tucson, AZ, USA \\ ${ }^{4}$ Department of Pathology, Center for Advanced Laboratory Medicine, University of California, San Diego, CA, USA \\ ${ }^{5}$ VA San Diego Healthcare System, San Diego, CA, USA \\ ${ }^{6}$ California Pacific Medical Center Research Institute, San Francisco, CA, USA \\ ${ }^{7}$ Sharp Mesa Vista Hospital, San Diego, CA, USA \\ ${ }^{8}$ Department of Psychiatry, Korea University College of Medicine, Seoul, Republic of Korea \\ ${ }^{9}$ Department of Psychology, Gyeongsang National University, Jinju, Republic of Korea
}

People called night owls habitually have late bedtimes and late times of arising, sometimes suffering a heritable circadian disturbance called delayed sleep phase syndrome (DSPS). Those with DSPS, those with more severe progressively-late non-24-hour sleep-wake cycles, and those with bipolar disorder may share genetic tendencies for slowed or delayed circadian cycles. We searched for polymorphisms associated with DSPS in a case-control study of DSPS research participants and a separate study of Sleep Center patients undergoing polysomnography. In 45 participants, we resequenced portions of 15 circadian genes to identify unknown polymorphisms that might be associated with DSPS, non-24-hour rhythms, or bipolar comorbidities. We then genotyped single nucleotide polymorphisms (SNPs) in both larger samples, using Illumina Golden Gate assays. Associations of SNPs with the DSPS phenotype and with the morningness-eveningness parametric phenotype were computed for both samples, then combined for meta-analyses. Delayed sleep and "eveningness" were inversely associated with loci in circadian genes NFIL3 (rs2482705) and RORC (rs3828057). A group of haplotypes overlapping BHLHE40 was associated with non-24-hour sleep-wake cycles, and less robustly, with delayed sleep and bipolar disorder (e.g., rs34883305, rs34870629, rs74439275, and rs3750275 were associated with $\mathrm{n}=37, \mathrm{p}=4.58 \mathrm{E}-09$, Bonferroni $\mathrm{p}=2.95 \mathrm{E}-06$ ). Bright light and melatonin can palliate circadian disorders, and genetics may clarify the underlying circadian photoperiodic mechanisms. After further replication and identification of the causal polymorphisms, these findings may point to future treatments for DSPS, non-24-hour rhythms, and possibly bipolar disorder or depression.

Psychiatry Investig 2014;11(4):345-362

Key Words Delayed sleep phase syndrome, Non-24 hour sleep-wake disorder, Bipolar disorder, BHLHE40, NFIL3, RORC.

\section{INTRODUCTION}

"Early to bed and early to rise, makes a man healthy, wealthy, and wise" according to Franklin's Poor Richard's Almanac. The economic advantages of being an early riser have been disputed $^{1}$ along with their wisdom. ${ }^{2}$ Many creative figures have

Received: April 15, 2014 Revised: April 18, 2014

Accepted: April 18, 2014 Available online: October 20, 2014

$\triangle$ Correspondence: Daniel F. Kripke, MD

Department of Psychiatry, University of California, San Diego, 8437 Sugarman Drive, La Jolla, CA 92037, USA

Tel: +1-858-222-2934, Fax: +1-858-554-8492, E-mail: dkripke1@san.rr.com

(c) This is an Open Access article distributed under the terms of the Creative Commons Attribution Non-Commercial License (http://creativecommons.org/licenses/by$\mathrm{nc} / 3.0$ ) which permits unrestricted non-commercial use, distribution, and reproduction in any medium, provided the original work is properly cited. anecdotally been late to rise or "night owls." On the other hand, late risers do tend to have more depression ${ }^{3,4}$ and to suffer a higher incidence of bipolar disorder, ${ }^{5-7}$ two of the most widespread illnesses disabling humanity. ${ }^{8}$ Among contemporary adolescents and college students, late risers tend to be poor school performers with social problems. ${ }^{9-12}$ A habit of arising late may reflect more than scorn for Franklin's philosophy, rebellion or indolence. In its severe form, being late to bed may constitute a biological disorder commonly termed "delayed sleep phase syndrome" (DSPS). Some contemporaries prefer "delayed sleep phase disorder," but both diagnostic formulations rest on nebulous criteria. ${ }^{13,14}$ Beyond mood disorders and occupational problems, delayed circadian phases have been associated with excess mortality.1,15 
"Larks" and "owls" are often distinguished by morningnesseveningness scales (MES) referencing behavior to time-ofday, disregarding whether sleep timing originates from a circadian system biologically tending to delay or whether late sleep hours may be appropriate to a person's preferences and social environments. The Basic Language Morningness Scale (BALM) employed in this research is one such semi-quantitative scale. ${ }^{16}$ DSPS and eveningness are closely related but not entirely overlapping phenotypes. Several studies have indicated that "eveningness" is partly inherited, often with dominant transmission. ${ }^{17-19}$ Environmental and social factors and puberty also play roles. ${ }^{20,21}$ Some studies suggest that genetic factors vary in penetrance according to a person's age. ${ }^{19}$ Fibroblast tissue cultures from those with extreme eveningness showed delaying circadian rhythms in vitro. ${ }^{22}$ Tissue from people with DSPS demonstrated a lengthened circadian period ${ }^{23}$ as do fibroblasts of bipolar patients. ${ }^{24}$ Somewhat like DSPS, bipolar depression may be associated with sleep delays and is strongly heritable. Thus, it is interesting to inquire whether polymorphisms in circadian system genes might partly explain these disorders.

Polymorphisms loosely associated with eveningness and delayed sleep phase have been reported, but these accounted for little phase variance and have not always been replicable. $^{25-28}$ Thus far, only a tiny portion of the genetic regulation of delayed sleep has been identified. A group of polymorphisms causing severe advanced sleep phase syndromes have been described, ${ }^{29}$ but these appear too rare to account for much of the population variability in morningness and eveningness. Therefore, there seems a need for an expanded search for polymorphisms in genes of the circadian system that might explain more biological aspects of delayed sleep.

We examined circadian genes in two cohorts with disturbed sleep (including some bipolar) and their controls and in an additional bipolar cohort, searching for polymorphisms associated with circadian phase delays.

\section{METHODS}

\section{Clinical sources}

Two independent clinical studies were used. Reports from early portions of these studies have previously appeared, ${ }^{4,30-33}$ as well as parallel analyses of associations of depression phenotypes with the same genetic material. ${ }^{34}$ To make our approach most accessible to readers, the investigative techniques will be presented even when description of specific methods may repeat portions of our previous reports.

\section{Participants}

From May, 2004 to March, 2011, volunteers for a study of "night owls" (DSPS) were recruited first by newspaper, radio, word-of-mouth, and internet advertising in the San Diego region, and later by internet advertising throughout the United States. Control volunteers who said their sleep was normal and that they were neither owls nor larks were likewise recruited, with targeting to approximately match the affected participants by age, gender, and ethnicity. Both delayed sleep phase and control participants signed written informed consent for the collection and use of their DNA and questionnaire responses, and they were paid for their participation, under supervision and ongoing approvals of the UCSD Human Research Protections Committee, otherwise known as an ethics committee (protocols 101294, 91633, 61397, 60999, 60014, 40211, 31645 , and 20026), and in compliance with the Helsinki Declaration of the World Medical Association. Participants also completed a series of questionnaires concerning their sleep timing and history of sleep disorders, especially delayed sleep phase symptoms, the Horne-Östberg ${ }^{35,36}$ and BALM ${ }^{16}$ morningness-eveningness scales, past medical and family history, psychiatric history including the Mood Disorder Questionnaire (MDQ), ${ }^{37}$ and also the QIDS-SR depression rating scale. ${ }^{38}$ Some of the DSPS participants underwent two weeks of wrist actigraphy. ${ }^{30}$ Assembling all this information including reported and measured timing of sleep, morningness-eveningness reports, reported distress, and lack of extraneous biological or social causes, the principal investigator and staff estimated their certainty that the participant suffered from a condition of primary biological origin making sleep undesirably late, indicating a "DSPS" phenotype. Participants contributed blood or saliva samples for DNA. Most of the samples were collected in Oragene saliva kits given or mailed to the participants (DNA Genotek Inc., Kanata, Ontario, Canada). There were 460 participants initially recruited as delayed and 458 recruited as controls, but not all could be included because of questionable phenotypes, unsatisfactory DNA samples, genotyping quality control, and missing data. The $\mathrm{n}=826$ initially available for analyses of genetic association were $67 \%$ female. The mean age was 38 years (range 22 to 82 ). Those of non-European ancestry were excluded from many analyses of genetic association, to limit genetic inflation due to sample stratification (Figure 1). The ancestry influence on allele frequencies is summarized in Supplement 1, Worksheets 8 and 9.

From June, 2006 to May, 2010, 1281 patients undergoing polysomnographic evaluation at the Scripps Clinic Viterbi Family Sleep Center were recruited for a study of genetic factors in sleep disorders. All patients age 21 years or older whom staff could conveniently contact and who were willing and able to consent were included. Written informed consent was obtained under the continuing approval and supervision of the Scripps Health IRB (ethics committee) protocols HSC-05- 


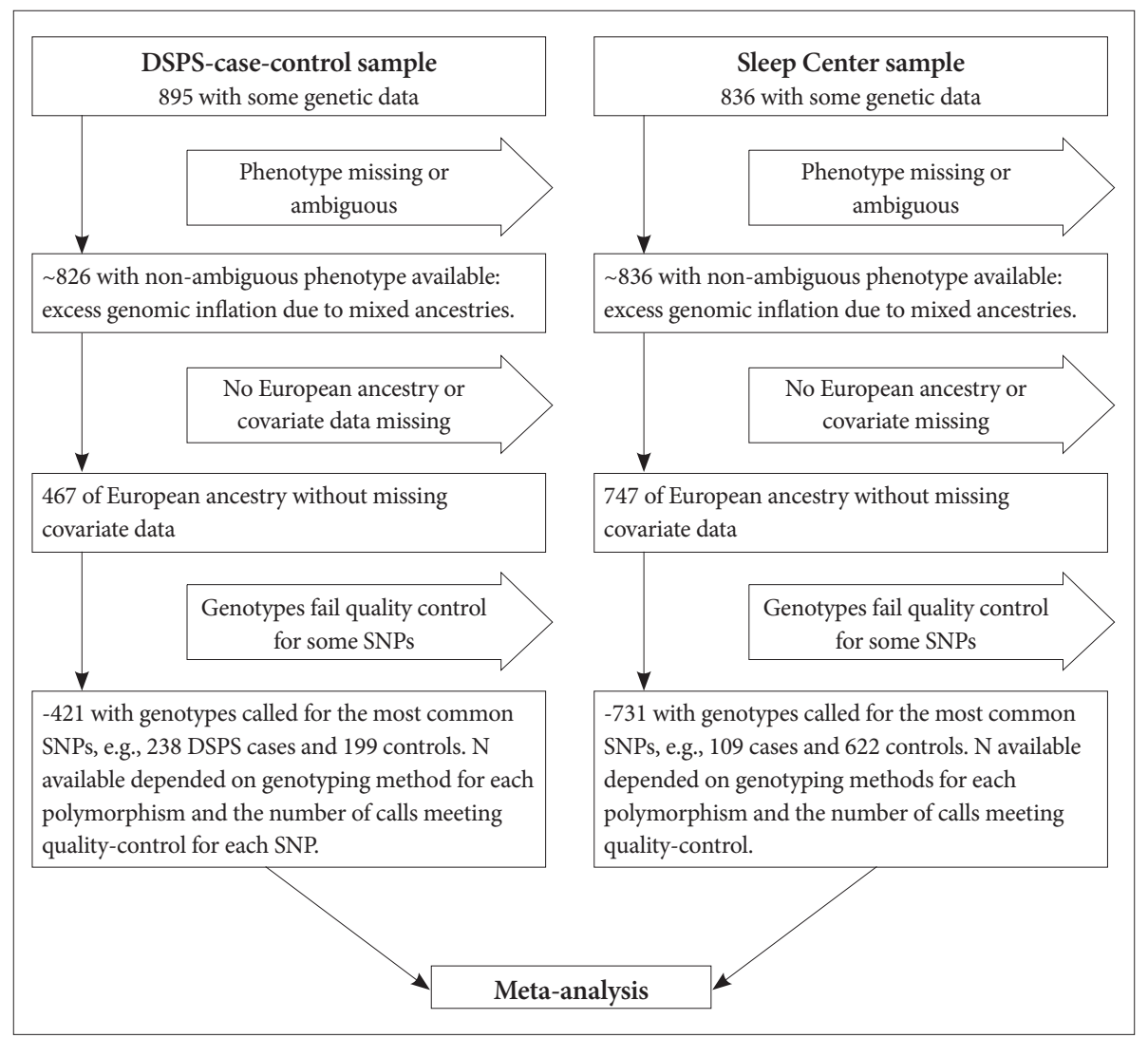

Figure 1. Flow chart of participant selection for the meta-analyses. Selection of samples for the main meta-analysis associating DSPS phenotype with SNPs is diagrammed. At progressive stages, samples were excluded if the DSPS phenotype determination was missing or ambiguous (e.g., a control volunteer who was rated possible DSPS). Because logistic regressions of the mixed-ancestry groups demonstrated excessive genomic inflation, those of non-European ancestry or uncertain ancestry were excluded from the meta-analysis. Samples were also dropped if covariate data were missing or if the genotype call for a particular SNP failed quality control. However, in supplementary analyses, we considered those with non-European ancestry (a mixture of African, Asian, Pacific Island, and Native American ancestry and mixed ancestries) as a group, and we considered all participants regardless of ancestry. DNA selections were similar for the DSPS-casecontrol phenotype and for the BALM quantitative phenotype.

2725 and IRB\# 05-707 and in compliance with the Helsinki Declaration, but patients were not paid for research participation. The patients' mean age was 57.6 years (range 21-96) and $64.8 \%$ were male. As part of a long clinical questionnaire inquiring about various symptoms relevant to sleep disorders, the BALM MES and the QIDS-SR self-rating depression scales were obtained. Clinician's sleep disorders diagnoses, including delayed sleep phase disorder (Code 327.31), were assembled. To best recognize a DSPS phenotype, those receiving a diagnostic code 327.31 or having BALM score $<28$ were classified as DSPS phenotype, a BALM $<28$ being suggested by our previous study. ${ }^{30}$ This was a more liberal BALM criterion than that used in our examination of pleiotropy with depression. ${ }^{34}$ Those patients not meeting DSPS criteria were utilized as controls. DNA was purified from saliva collected in Oragene sampling kits, but it has not yet been possible to assay genotypes in the last portion of the clinical sample. Those labeled DSPS included 14.9\% of 731 patients (109 DSPS) of European ancestry usable in Sleep-Center DSPS phenotype analyses and with adequately assayed genotypes.

\section{DSPS diagnoses}

In a representative population sample of San Diego adults 40-64 years of age, Ando et al. found that 10.9\% reported trouble falling asleep at least 3 times/week, 16.9\% reported trouble waking up in the morning at least 3 times/week, and $3.1 \%$ reported both. ${ }^{39}$ Though it is unlikely that such high percentages should be classified as having DSPS, there is as yet no validated basis for specifying the strength or validation of complaints that should be required for diagnosis. Ando et al. reported that average actigraphic bedtimes were $10 \mathrm{~min}$. later than reported bedtimes, and actigraphic wake-up times were $22 \mathrm{~min}$. later, with the participants awakening $45 \mathrm{~min}$. later on weekend mornings. ${ }^{39}$ Sleeping late on weekend mornings may result from a variable combination of delayed sleep propensities, late-night social and recreational opportunities on weekends, and a build-up of sleep-debt during the work week. Clinical definitions have not yet evolved a practical method- 
ology for accommodating the discrepancies between subjective and objective sleep timing and between week-day and weekend habits. For these studies, we have used the historical term 'delayed sleep phase syndrome" (DSPS) to avoid complying with the nebulous contemporary clinical criteria for "delayed sleep phase disorder" that depend on discerning "a delay in the phase of the major sleep period in relation to the desired sleep time and wake-up time," also requiring that "when allowed to choose their preferred schedule, patients will exhibit normal sleep quality and duration."13,14 It is often difficult to judge and document when such misalignment of sleep propensity is sufficient for diagnosis, or when social factors, personal preferences, or psychophysiological disturbances are the main factors in a person's sleep timing. Also, even when the endogenous circadian phase has played a crucial role in establishing a person's sleep time preferences, some people adapt to a late endogenous circadian sleep propensity so well that they no longer would prefer an earlier sleep time. Other people with a late endogenous circadian phase are so upset by lying in bed for long hours trying to sleep that they suffer psychophysiological disturbances arising from adverse habit development and impaired sleep efficiency, even when they retire at the time of their optimal sleep propensity, so that their sleep is no longer symptom-free at any time of day. Lacking logical, specific, and validated clinical diagnostic criteria, we have frankly relied on the investigator's synthesis and balance of information suggesting either biological misalignment or sleep later than the normal range to judge when a circadian disorder of biologic origin produces unwelcome delay. The degree of certainty that a person had a biologic disorder of delay was not based upon one specific set of criteria but on a judgment synthesizing reported and preferred sleep times (both current and past), the Horne-Östberg and BALM scales for morningness-eveningness, sleep complaints, sleepiness and fatigue complaints, confounding conditions, and various details of past history. Comorbidity with depression or mania did not prevent DSPS classification unless mood disorder symptoms appeared first and appeared to cause the sleep disturbance.

\section{Certainty of DSPS diagnoses}

In the DSPS-case-control sample, DSPS phenotypic cases were defined as previously described as absolutely certain, certain, and questionable, or not DSPS, and controls were included only if there was no suspicion of DSPS, though a few controls with possible ASPS were included. ${ }^{30}$ It was found that combining the three levels of DSPS certainty yielded the most statistically-robust genetic associations, probably because of the greater numbers available for the regressions of a broader phenotype. In the Sleep Center sample, patients were scored as having DSPS phenotype if a DSPS or DSPD diagnosis was recorded in the medical record, and/or if the BALM score was $<28$. The BALM alternate criterion was needed because clinicians sometimes omitted making a DSPS diagnosis when the primary diagnostic focus was sleep apnea, WillisEkbom disease, etc. Having studied the data further, we used this looser BALM criterion for DSPS yielding almost twice as many DSPS cases as in of our previous report on the same data wherein the BALM criterion used was $23 .^{34}$ It was surprising that the change in criteria made substantial alterations in which SNPs were nominally associated with DSPS in the Sleep Center sample, particularly reducing the nominal association of GSK3B SNPs. To give another interesting example, MTNR1A rs13131052 was more strongly associated with DSPS than NFIL3 rs2482705 in some logistic regressions of Sleep Center data using the older DSPS phenotype form. As prospectively predicted, in both the DSPS-case-control and Sleep Center data sets, the strongest associations to DSPS and BALM were generally found restricting the sample to participants of European ancestry $<60$ years of age, using dominant models.

\section{Recognizing non-24-hour sleep-wake rhythms}

To evaluate the presence of non-24-hour rhythms, actigraphs were evaluated (by DFK) for those DSPS cases who had completed usable actigraphy $(n=380)$, and additional clinical data were considered when available for both those recruited as DSPS cases and controls. Based on 2 weeks or less of continuous actigraphy, the recognition of a non-24-hour component was often uncertain, both because a full $360^{\circ}$ progression of the non-24-hour component can almost never be visualized within 2 weeks and because such components are often masked by social and environmental synchronizers. In some cases, questionnaire data about various sleep symptoms and previous treatment could assist recognition of these phenomena or longer actigraphic or sleep log records were available. Since any data on such cases are rare and require years to collect, to best exploit the data available, we scored participants (blind to genotyping) for non-24-hour rhythms as 3) definite, 2) likely, 1) possible, or 0) no evidence. Apart from one Sleep Center patient who underwent actigraphy for a non-24-hour rhythm, adequate determination of non-24-hour rhythms in the Sleep Center sample could not be done, nor was this determination possible for the controls of DSPS cases (who did not undergo actigraphy) or bipolar patients recruited from clinical sources. Neither non-24-hour rhythms nor bipolar disorder were the primary focus of this research, and neither disorder is easily identified by the methods we used except in the most extreme cases. The majority of those affected might exhibit only partial penetrance leading to serious 
insensitivity in our ascertainment. Technologic innovations will make this ascertainment more practical in the future.

\section{Identifying bipolar disorder}

Although the presence of unipolar depression or bipolar disorder were only incidental foci of the studies, the DSPScase-control sample completed the Mood Disorder Questionnaire (MDQ), a screening instrument for bipolar I and bipolar II disorders with 0.28 sensitivity and 0.97 specificity. ${ }^{37}$ Since sensitivity is improved by relaxing the criteria at some loss of specificity, participants not meeting strict criteria were scored 1) possible, 2) likely, or 3) definite bipolars by weighing a number of factors in addition to MDQ scores, such as how they answered explicit questions about having had mania or bipolar disorder, about hospitalizations, and about medications they had taken, their comments when interviewed, and other information when offered. This bipolar scoring was done by DFK blind to genotypes. Of 918 participants in the DSPScase-control study (not all of whom could be genotyped) plus 8 bipolar patients added to the sample from clinical sources, only 11 were scored definite bipolars, 31 likely, and 43 possible, with the remainder scored 0 for no evidence of bipolar disorder. As previously reported, the rate of bipolar disorder was greater among DSPS cases than their controls. ${ }^{30}$ In the Sleep Center study, neither the MDQ nor other relevant questionnaire items for bipolar disorder were available, but with access to the electronic medical records (which generally did not focus on psychiatric diagnoses), 10 patients were found with bipolar diagnoses, among which 8 had usable genotypes and were of European ancestry.

\section{Genotyping}

To identify polymorphisms relatively unique to DSPS, which might not have been previously reported, portions of 15 circadian genes in up to 45 selected DNAs (mainly from DSPS cases) were resequenced with Sanger capillary sequencing using ABI 3730 technology ${ }^{40}$ These genes were ARNTL, BHLHE40, BHLHE41, CLOCK, CRY1, CRY2, CSNK1D, CSNK1E, DBP, NFIL3, NPAS2, NR1D1, PER1, PER2, and PER3. Although the 36 samples from DSPS cases and controls resequenced proved too few for DSPS case-control contrasts, resequencing of BHLHE40 produced observations relevant to non-24-hour circadian phenotypes and bipolar disorder.

As will be described in the results, by inspecting the resequenced genotypes, we unexpectedly observed a group of haplotypes forming a distinctive allele pattern in BHLHE4O. In BHLHE40, a participant's DNA was considered to display this group of haplotypes when at least two of the 18 linked minor alleles were observed, and a SNP was judged to be a component of this group of haplotypes when the minor alleles were observed in at least two participants together with other components of the group of haplotypes.

To obtain data from more DNAs and additional polymorphisms, an expanded genotyping effort was organized using a custom Illumina Golden Gate assay targeting 768 SNPs selected from genes thought crucial in circadian rhythm regulation and including a few SNPs implicated in sleep disorders. ${ }^{41}$ Genotyping was performed following the assay manufacturer's instructions. Genotypes were clustered within GenomeStudio using all samples with $>95 \%$ call rates. SNPs with call rates $<90 \%$ or heterozygote frequencies $>65 \%$ after re-clustering were removed. Cluster positions for each SNP were manually inspected and edited if necessary. The final Illumina assay dataset for the Sleep-Center patients genotyped 630 SNPs (average call rate 99.7\%). In addition, 41 SNPs selected as ancestry-informative markers (AIMs) were genotyped with Sequenom and SNPlex assays. ${ }^{42}$ Various polymorphisms including the PER3 VNTR (rs57875989) were explored with supplemental Taqman assays. After screening for genotyping quality control, adequate heterozygosity, and HardyWeinberg equilibrium, the DSPS case-control Illumina assay sample included 667 circadian-sleep-gene-related polymorphisms available for linear regression including 27 of the ancestry-informative markers. The ancestry-informative markers were used in combination with patient self-reports of ancestry to select those of European origin, when inclusion of those with nonEuropean ancestry yielded an excessive genomic inflation factor. The non-Europeans were a diverse group with African, Asian, Pacific Island, or Native American ancestry, as well as mixed ancestries. Tables in Supplement 1 provide detailed listings of Illumina assay genes, SNPs, and statistical outcomes.

\section{Association analyses}

The associations of the sample polymorphisms with the morningness-eveningness scales were determined using the linear regression module of PLINK ${ }^{43}$ with a prospectively-selected dominant model, limiting the samples to those of European origin and controlling for covariates of age and gender. In addition, genetic background was estimated using multidimensional scaling (MDS) of the AIMs, and the top 2 MDS components ${ }^{42}$ were used as covariates in the linear regression model to minimize genomic inflation. In some analyses, the samples were also limited to those <age 60 years, because associations with the MES scales proved stronger. In addition, because our previous analysis suggested five factors composing the revised Horne-Östberg scale, ${ }^{33}$ the association of each factor with genetic markers was determined independently by linear regression in the DSPS-case-control sample. For polymorphisms of interest located in the non-autosomal re- 
gion of the $\mathrm{X}$ chromosome, males and females were analyzed independently. Then PLINK meta-analyses were used to combine results of both DSPS-case-control and Sleep Center studies. Significance was assessed with Bonferroni adjustments for testing multiple polymorphisms. Adjustments for multiple comparisons were also checked by computing false-discovery statistics, ${ }^{44}$ but these were sufficiently consistent with the Bonferroni outcomes to make their separate presentation superfluous. The fixed-effects $\mathrm{p}$ values have been listed, but for SNPs where Cochrane's $Q$ statistic was $Q<0.05$ indicating significant heterogeneity in the fixed-effects models, though the random-effects $\mathrm{P}(\mathrm{R})$ was $\mathrm{p}>0.05$, the meta-analysis value for the SNP was considered NS. For candidate-gene analyses, PLINK set-based tests were computed on linear regressions using default parameters of the -set command $\left(\mathrm{r}^{2}=0.5, \mathrm{p}<\right.$ 0.05 , maximum number of SNPs=5) and 10,000 permutations. For contrasts of the DSPS phenotype in the Sleep Center sample, similar logistic regressions and genewise -set tests were computed.

\section{RESULTS}

\section{Alleles in BHLHE40 associated with non-24-hour sleep-wake cycles}

We used Sanger resequencing of 15 gene regions to find polymorphisms that might be related to DSPS and phase delay, using 45 DNA samples from research participants with severe DSPS syndromes, non-24-hour rhythms, bipolar disorder, or controls. We identified over 700 polymorphisms in genes of the circadian system..$^{40} \mathrm{~A}$ grouping of associated alleles was retrospectively observed in BHLHE40 (previously designated $\mathrm{DEC} 1^{45}$ or BHLHB2-see EF015895.1). ${ }^{40}$ The pattern of minor alleles encompassed 17 variably-linked SNPs and one indel among the 29 polymorphisms detected in the 5 ' promoter, 5 ' UTR, introns, exons, 3'UTR, and near 3' downstream region (Figure 2). Haploview ${ }^{46}$ Hapmap 3 data (which include 4 SNPs of the associated allele group) suggest that in Europeans, this loose pattern is interlinked with a network of strong linkage disequilibrium blocks spanning approximately $80 \mathrm{~kb}$ both $3^{\prime}$ and 5 ' to BHLHE4O. The samples available in the 1000 Genome Pilot 1 suggest a somewhat more limited extent of linkage disequilibrium (Figure 3).

In a severe variation developing from DSPS, sleep and wake arrive later and later, day after day, until a non-24-hour rhythm in sleep-wake may become recognizable (Figure 4). For 37 of the resequenced DSPS participants, the probability of a non24-hour circadian component could be estimated from actigraphy, besides one bipolar patient known to have had a 21.8 hour non-24-hour rhythm in early adulthood. ${ }^{47}$ Of the 38 , three definitely had non-24-hour circadian rhythms, and

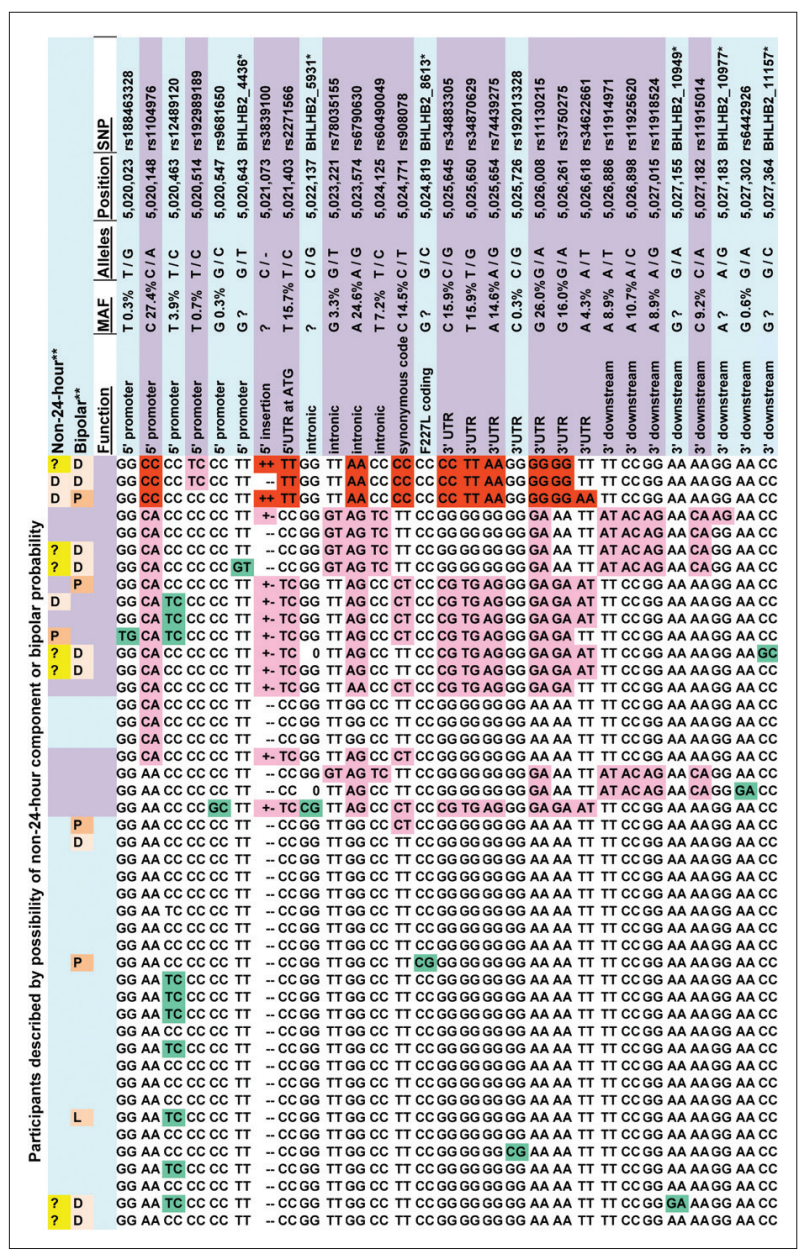

Figure 2. Patterns of associated BHLHE4O SNPs with phenotypes. Rows represent each of the 45 participants resequenced (8 bipolars and 37 DSPS), and columns describe the SNPs. Position is the chromosome-3 base position from assembly $\mathrm{GRCH} 37$ / hg19. Alleles list the minor allele first, as indicated in the forward DNA strand, and MAF shows the minor allele frequency listed in the UCSC browser 7/6/2013. For the associated SNP pattern: Red shading highlights a minor allele homozygote, and pink shading highlights a minor allele heterozygote for the associated pattern of alleles. Green shading highlights a SNP that was not inferred to be one of the 18 interlinked polymorphisms. Purple highlights the associated allele group (top of columns) and participants with $\geq 2$ such alleles (left columns). *for specification of SNPs without "rs" designations (not appearing in dbSNP) see http://www.ncbi. nIm.nih.gov/nuccore/EF015895, **regarding non-24-hour sleepwake components or bipolar disorder, symbols in columns 2-3 for a participant indicate. P: possible, L: likely, D: definite, or ?: undetermined.

one was judged to possibly display a non-24-hour circadian rhythm component. Two homozygous for the associated-minor-allele pattern definitely had non-24-hour rhythms, and the "possible" non-24-hour participant plus the other definite example were heterozygous ( $\mathrm{p}=0.004$, exact 2 -sided test) (Figure 2, Table 1). The Spearman nonparametric rank order correlation associating the probability of a non-24-hour rhythm (scored 3, 2, 1, or 0) with the number of distinctive-pattern alleles per participant was $\mathrm{R}_{\mathrm{s}}=0.492$ (exact significance $\mathrm{p}=0.001$, 


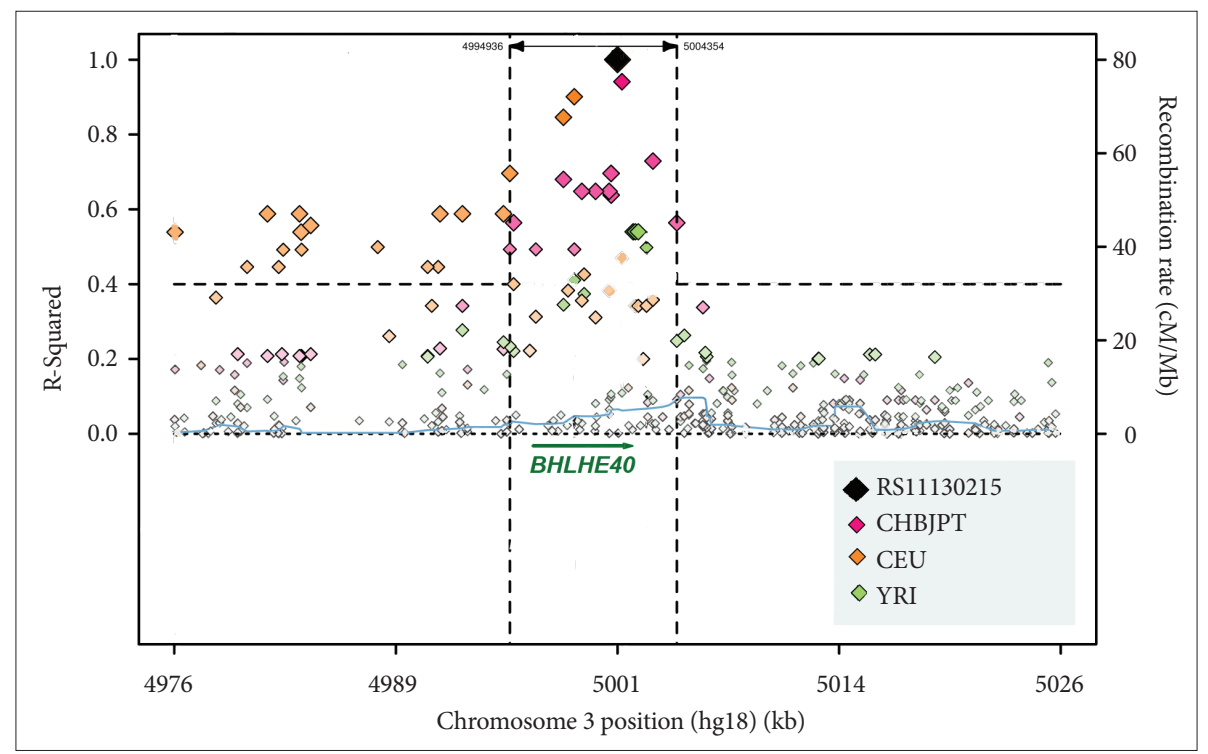

Figure 3. Linkage disequilibrium of BHLHE40 by ancestry. Linkage $\left(r^{2}\right)$ of $B H L H E 40$ SNP rs 11130215 is shown as an example for the haplotype group, compiled and edited from SNAP plots ${ }^{48}$ for CEU, CHBJPT, and YRI ancestry groups within the 1000 Genome Pilot 1 data base (hg18 locations). The range plotted is from 25K 5' to the SNP (just at the limit of CEU significant linkage) to 25K 3' to the SNP. The sample with CEU ancestry had far stronger 5' linkage than the Asian or YRI samples, but linkage dropped sharply within a few thousand nucleotides downstream of the 3' UTR in all groups. BHLHE40 is a small gene (green arrow). Its protein binds to E-box transcription factor binding sites of several circadian genes, deactivating promoter activity by competitive inhibition. ${ }^{45,56}$ BHLHE40 forms heterodimers with BHLHE41. BHLHE40 transcription is enhanced by light pulses, probably due to a CRE-responsive element in its promoter. According to the Scripps Genome Adviser, ${ }^{49}$ several of the $B H L H E 40$-associated alleles may influence transcription binding sites of the adjacent gene.

one-tailed). Considering the estimated probability of a non24-hour rhythm, a linear regression model for all ethnicities (genomic inflation factor=1) found association for 9 of the linked SNPs of BHLHE40, including rs34883305, rs34870629, rs74439275, and rs3750275 (all n=37, Beta $=0.99, \mathrm{SE}_{\text {Beta }}=0.18$, $\mathrm{p}=4.58 \mathrm{E}-09$, Bonferroni $\mathrm{p}=2.95 \mathrm{E}-06)$ as well as $\mathrm{rs} 908078$ ( $\mathrm{p}=$ 1.53E-07, Bonferroni $\mathrm{p}=9.87 \mathrm{E}-05)$, rs11130215, rs1104976, rs2271566, and rs6790630 (all Bonferroni significant). See also Table 2.

\section{Ancestry factors in BHLHE40 associated alleles}

Regarding BHLHE40, only one of 15 resequenced DNAs with the pattern of associated alleles was judged non-European by ancestry-informative markers and self-reported ancestry, whereas 5 of the 30 with none of the associated alleles were typed non-European or of mixed origin. Nevertheless, 1000 Genomes Browser data for 100-200 subjects per group indicate higher frequencies of BHLHE40 pattern alleles among Africans (e.g., averaging 20.8\% among YRI and 17.2\% among LWK in contrast to $10.6 \%$ among CHB and $10.5 \%$ among JPT and in contrast to $8.7 \%$ among CEU and $11.4 \%$ among TSI). This raises the possibility that the associated allele pattern might derive from an ancient haplotype of African origin that gradually evolved and became largely or entirely replaced in most BHLHE40 genotypes of Asian and European ancestry. SNAP analyses of linkage disequilibrium ${ }^{48}$ in 1000 Genome Pilot 1 data sets indicated that CEU Europeans had a broader region of linkage disequilibrium than CHBJPT Asians and a much broader region of linkage disequilibrium than YRI Africans (Figure 3), perhaps suggesting that the common-allele pattern among Europeans is of more recent origin. Among the Europeans, linkage $\mathrm{r}^{2}$ was as high as 0.67 as far as 27K 5' of BHLHE40, but substantial linkage with $r^{2}>0.40$ did not extend 3 ' beyond the 3' UTR. As might be inferred from Figure 2, rs908078 was not as closely linked with other SNPs within BHLHE40 as some of the other SNPs, nor was it as strongly linked to the 5 ' promoter. The weaker association of rs 908078 with non-24-hour rhythms than other BHLHE40 SNPs may be a clue that linked alleles in the 5 ' promoter region are important to the phenotype associations observed.

\section{Non-24-hour cycle patient characteristics}

All our examples of possible non-24-hour rhythms had cycles longer than 24.0 hours, except for the one bipolar participant who definitely had a 21.8 hour rhythm in early adulthood, ${ }^{47}$ not observed when she was restudied by actigraph over 20 years later. Besides two chromosomes with most of the BHLHE40 grouped alleles, this participant had a unique PER2 polymorphism, ${ }^{40}$ previously described as PER2_21007 (http://www.ncbi.nlm.nih.gov/nuccore/EF015905) but so far as we have learned, never described elsewhere. It is possible that this unique polymorphism explains her unusual non24-hour cycle. Since NCBI has not assigned this polymorphism a dbSNP number or description, as reference genomes 


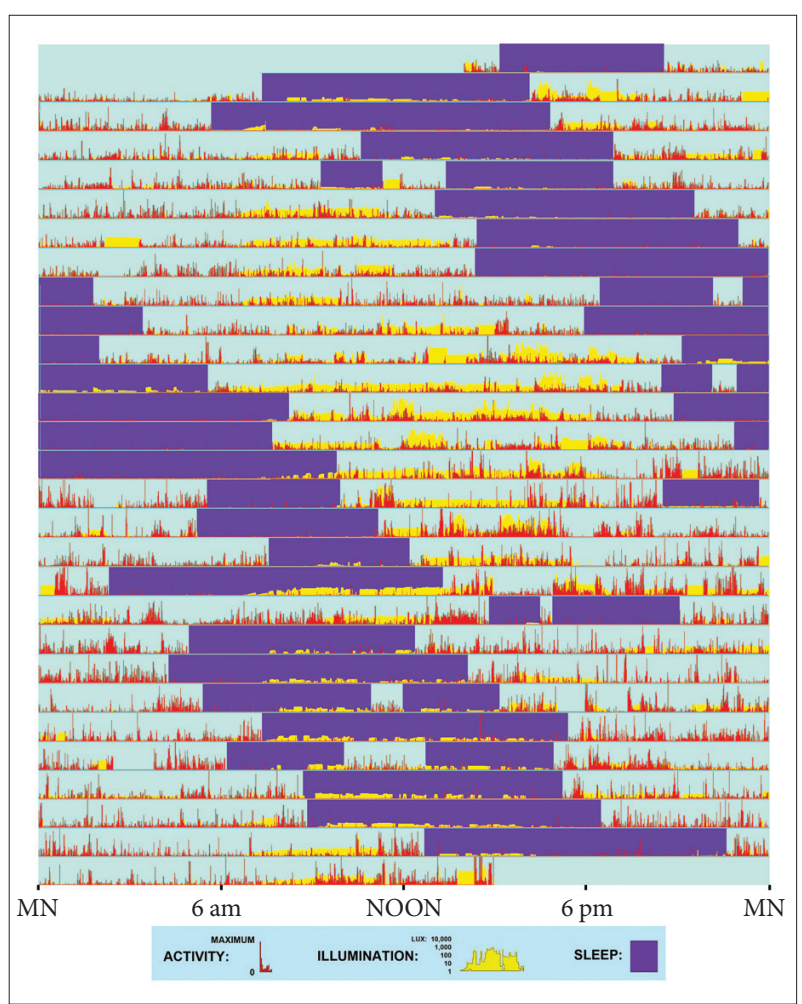

Figure 4. Actigraphic record provides an example of a non-24hour sleep-wake rhythm. This patient wore a wrist activity-light monitor for 4 weeks (starting and ending about 2 PM). On each day (consecutive horizontal lines), the red vertical bars show relative wrist activity, indicating wakefulness. Purple rectangles highlight intervals of predominant inactivity, inferred as sleep with assistance of the patient's sleep log. Vertical yellow bars display the patient's illumination surroundings, plotted on a semi-logarithmic scale of lux (from 1 lux, e.g., full moonlight to 10,000 lux, e.g., bright sunlight.) Note that on many days, the patient experienced little or no daylight. This patient habitually retired to bed an hour or two later each day and awakened correspondingly later ( $25.4 \mathrm{hr}$. cycle.) His sleep drifted around the clock, with only minimal pattern irregularities suggesting interactions with the environment. Brief intervals when the actigraph was removed for bathing were not edited.

are revised, this $\mathrm{T}<\mathrm{C}$ polymorphism might best be identified by its context on the reverse DNA strand:

GGATCCCACATGTGCTGGGACCATCATGAAGCTG

\section{GCCTCATAACAGGGCT}

\section{$\mathrm{Y}$}

\section{CCTAATCCTTCCCATCTTCTTCCTCCAGCGTA ACCCCCAGGCAGCCCCCA}

\section{BHLHE40 alleles and bipolar disorder}

All 3 participants homozygous for the BHLHE40 linked allele pattern were judged to have possible or definite bipolar disorder. Six of the 9 definite bipolars had at least one chromosome with the associated allele pattern, as compared to 9 of 36 who were not bipolar ( $\mathrm{p}=0.042$, two-tailed Fisher Exact Test). Considering the nonparametric correlation of a participant's number of alleles with the associated pattern and the likelihood of bipolar disorder (ranked 0 for improbable, 1 for pos-
Table 1. The minor allele pattern vs. non-24-hour rhythm possibility

\begin{tabular}{lcclc}
\hline \multirow{2}{*}{$\begin{array}{c}\text { Estimated } \\
\text { rhythm }\end{array}$} & \multicolumn{3}{c}{$\begin{array}{c}\text { N chromosomes with } \\
\text { haplotype alleles }\end{array}$} & \multirow{2}{*}{ Total } \\
\cline { 2 - 4 } & 0 & 1 & 2 & \\
\hline 24-hour rhythm & $24(71 \%)$ & $10(29 \%)$ & $0(0 \%)$ & $34(100 \%)$ \\
Possible non-24 hr. & $1(0 \%)$ & $1(100 \%)$ & $0(0 \%)$ & $1(100 \%)$ \\
Definite non-24 hr. & $0(0 \%)$ & $1(33 \%)$ & $2(67 \%)$ & $3(100 \%)$ \\
Total & $24(62 \%)$ & $12(32 \%)$ & $2(5 \%)$ & $38(100 \%)$ \\
\hline
\end{tabular}

The columns show the numbers of the resequenced participants with 0,1 , or 2 chromosomes with the minor allele pattern, that is, homozygotes for the common alleles, heterozygotes, and homozygotes for the minor allele pattern. Rows tabulate participants estimated to have a clear 24-hour sleep-wake rhythm by actigraphy or a possible or definite non-24-hour sleep-wake rhythm

Table 2. Non-24-hour components associated with BHLHE4O rs908078 alleles

\begin{tabular}{lcccc}
\hline \multirow{2}{*}{$\begin{array}{c}\text { Non-24-hour } \\
\text { component }\end{array}$} & \multicolumn{2}{c}{ Rs908078 number of C alleles } & \multirow{2}{*}{ Total } \\
\cline { 2 - 4 } & 0 & 1 & \multicolumn{1}{c}{2} & \\
\hline None & $30(77 \%)$ & $9(23 \%)$ & $0(0 \%)$ & $39(100 \%)$ \\
Possible & $7(47 \%)$ & $8(53 \%)$ & $0(0 \%)$ & $15(100 \%)$ \\
Probable & $4(100 \%)$ & $0(0 \%)$ & $0(0 \%)$ & $4(100 \%)$ \\
Definite & $1(25 \%)$ & $1(25 \%)$ & $2(50 \%)$ & $4(100 \%)$ \\
Total & $42(68 \%)$ & $18(29 \%)$ & $2(3 \%)$ & $62(100 \%)$ \\
\hline
\end{tabular}

All three participants with definite non-24-hour cycles (Figure 1) were heterozygous for the $\mathrm{C}$ (minor) allele of rs908078, as was the participant with a possible non-24-hour component and 6 with delayed 24-hour rhythms. None of 28 participants out of 45 without the $\mathrm{C}$ allele had any suspicion of a non-24-hour component $\left(R_{s}=0.560, p=0.006\right)$, though some such were seen with extended genotyping. In this table showing the larger group of all our DSPS cases for whom we had actigraphic judgments regarding non-24hour circadian activity components and rs908078 Illumina genotyping, the minor $\mathrm{C}$ allele and suspected non-24-hour components were associated, with Spearman correlation of $\mathrm{R}_{\mathrm{s}}=0.274$ and $\mathrm{p}=0.033$; moreover, the exact significance of the Pearson ChiSquare of $36.873(\mathrm{DF}=6)$ was $<0.001$. Because the rs 908078 minor $\mathrm{C}$ allele was found in only 10 of the 18 DNAs in which the $B H L$ $H E 40$ pattern of alleles were found, the rs $908078 \mathrm{C}$ allele served as a partial marker for the group of haplotypes, but was not the bestassociated marker related to non-24-hour components

sible, 2 for likely, or 3 for definite), Spearman's rho was 0.32 (exact significance $\mathrm{p}=0.034$, two-tailed). When linear regression of the BHLHE40 alleles against the bipolar score were computed, 10 of the 17 SNPs of the minor allele pattern were significantly associated with $\mathrm{p}<0.05$, but in this small data set, none of these associations reached Bonferroni criteria. A PLINK ${ }^{43}$ genewise-set test for overall significance of the BHLHE40 alleles for bipolar disorder was not significant. There was little or no association of bipolar disorder with non-24-hour sleepwake cycles (Table 3).

\section{Rs 908078 and bipolar disorder and DSPS}

From BHLHE40, rs908078 was the only allele-pattern-as- 
Table 3. Bipolar disorder likelihood vs. non-24-hour component likelihood

\begin{tabular}{|c|c|c|c|c|c|}
\hline & \multicolumn{4}{|c|}{ Likelihood of non-24-hour circadian component } & \multirow{2}{*}{ Total } \\
\hline & None & Possible & Probable & Definite & \\
\hline NOT bipolar & $21(70 \%)$ & $8(27 \%)$ & $0(0 \%)$ & $1(3 \%)$ & $30(100 \%)$ \\
\hline Possible bipolar & $8(80 \%)$ & $1(10 \%)$ & $0(0 \%)$ & $1(10 \%)$ & $10(100 \%)$ \\
\hline Probable bipolar & $3(60 \%)$ & $1(20 \%)$ & $1(20 \%)$ & $0(0 \%)$ & $5(100 \%)$ \\
\hline Definite bipolar & $0(0 \%)$ & $0(0 \%)$ & $0(0 \%)$ & $2(100 \%)$ & $2(100 \%)$ \\
\hline Total & $32(68 \%)$ & $10(21 \%)$ & $1(2 \%)$ & $4(9 \%)$ & $47(100 \%)$ \\
\hline
\end{tabular}

Blind estimations of whether or not a participant was bipolar were tabulated versus blind estimations of whether or not a non-24-hour circadian sleep-wake component was likely, among those with DSPS phenotypes for whom both ratings were available. Among the 38 participants whose DNA was resequenced and whose wrist activity was recorded, there was no significant association between suspected bipolar disorder and a suspected non-24-hour rhythm. In our larger set of DSPS cases, the exact test (two-sided) relating non-24-hour possibility and bipolar possibility had a Pearson Chi-Square of 32.538 with $\mathrm{DF}=9, \mathrm{p}=0.005$, but this appeared to have almost entirely reflected the two cases homozygous for the rare allele who had non-24-hour rhythms, because the non-parametric correlation was only $\mathrm{R}_{\mathrm{s}}=0.182$ (NS)

sociated SNP successfully genotyped in the Illumina assay, though rs11130215 was imputed. We could not demonstrate significant association of rs908078 or rs11130215 with bipolar disorder in this larger sample $(\mathrm{n}=463-466)$, but the bipolar probability scores in homozygotes or heterozygotes for the distinct grouping of BHLHE 40 minor alleles averaged about 3 times the probabilities for homozygotes with the common alleles. Meta-analysis demonstrated that rs 908078 was nominally associated with the DSPS phenotype (OR=1.39, $\mathrm{p}=$ 0.034 ) and with less morningness (greater eveningness) on the BALM scale (Beta $=-1.81, \mathrm{p}=0.018$, shown in Supplement 1 Worksheets 1 \& 2). Rs908078 associations did not meet Bonferroni criteria. In the DSPS affected cases, the rs 908078 minor-allele homozygotes and heterozygotes, and the major allele homozygotes were distributed as 6/82/150, with observed MAF (minor allele frequency) $=0.3445$, whereas among unaffected participants, the distribution was 5/45/149 with $\mathrm{MAF}=0.2261$. In the Sleep Center sample, the MAF averaged 0.26 with DSPS affected having only $0.016 \%$ more minor alleles than controls unaffected. These larger samples support the role of rs 908078 (and by inference, the BHLHE40 associated alleles) in delayed sleep phase, as well as in non-24-hour rhythms. According to the Scripps Genome Adviser, ${ }^{49}$ rs 908078 is a synonymous coding SNP which creates an ESE site, which may promote splicing.

\section{Two loci associated with DSPS morningness- eveningness}

To gather more data, the DSPS cases and controls and the independent sample of patients undergoing polysomnography at a Sleep Center were genotyped with a custom Illumina assay chip, probing 768 SNPs in 41 circadian and sleep-related genes. Logistic regressions of the DSPS phenotype with Illumina SNP genotypes were examined separately in the DSPScase-control sample and in the Sleep-Center sample, followed by meta-analysis combining the two samples (Supplement 1).

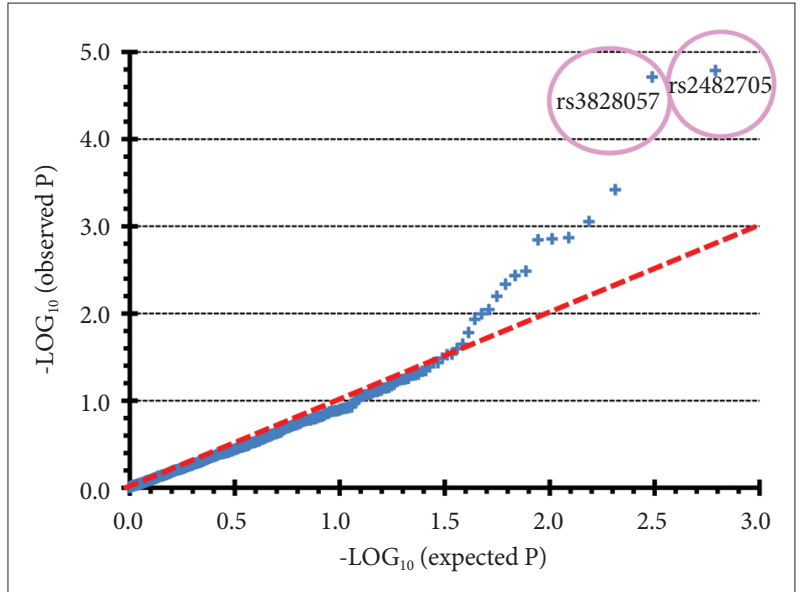

Figure 5. Q-Q plot for the meta-analysis of the DSPS phenotype. This quantile-quantile plot shows SNP association $p$ values from the meta-analysis of the DSPS phenotype in the combined DSPScase-control and Sleep Center data sets. See the first worksheet of Supplement 1 for a listing of these $p$ values. Quantile-quantile plots can be useful in visualizing the import of SNPs associations by comparing the distributions of nominal $p$ values with the random expectation, and they are useful in appraising possible confounding from sample stratification. The ordinate plots the observed probability $(P)$ values on a minus $L O_{10}$ scale, and the abscissa represents the expected probabilities of a random distribution on a comparable scale. The dashed red line highlights the random expectation of $\mathrm{P}$ statistic distributions. Pink circles highlight the two Bonferroni-significant SNPs. Note that the next 5-10 nominally-significant SNPS were sufficiently elevated above the expectation to suggest possible association.

As prospectively anticipated, ${ }^{18}$ the dominant model gave more robust results. In the meta-analysis of the two samples, 25 of 616 SNPs were associated with a DSPS phenotype with nominal $\mathrm{p}<0.05$, but only two were significant after Bonferroni correction (Figure 5).

In NFIL3 (previously designated E4BP4), rs2482705 was significantly associated with the DSPS phenotype by metaanalysis $(\mathrm{OR}=0.47$, nominal $\mathrm{p}=1.63 \mathrm{E}-05$, Bonferroni-correct$\mathrm{ed} \mathrm{p}=0.010$ ). Association of $\mathrm{rs} 2482705$ was only significant in the DSPS-case-control sample (Table 4 and Supplement 1), but including the Sleep-Center patients somewhat strength- 
ened the meta-analysis, since the Sleep-Center rs2482705 association approached nominal significance $(\mathrm{p}=0.052)$, and the odds ratios in the two studies were rather consistent. Examining imputed SNPs, rs2482705, rs2440592, and rs2482359 had identical associations with DSPS, for linkage extends widely (Figure 6). Six of the 13 SNPs examined in NFIL3 meta-analysis had $\mathrm{p}<0.05$ association with $\mathrm{OR}$ of 0.47 to 0.67 . The PLINK $^{43}$ genewise --set test for NFIL3 in the DSPS-case-control sample yielded $\mathrm{p}=0.0037$ (Supplement 1 ). Note that the common $\mathrm{G}$ allele was associated with delayed sleep, whereas the minor A allele was associated with sleep being less de- layed (i.e. relatively more advanced).

Likewise, rs3828057 in RORC was associated with the DSPS phenotype in the meta-analysis (with $\mathrm{OR}=0.51$, nominal $\mathrm{p}=1.94 \mathrm{E}-05$, Bonferroni $\mathrm{p}=0.012$ ). Moreover, this SNP was nominally significant in both the Sleep Center and DSPS-casecontrol samples, and RORC was significant in the Sleep-Center genewise -set test $(\mathrm{p}=0.0008)$ (Supplement 1$)$, but strong linkage disequilibrium extends beyond RORC (Figure 7).

PLINK linear regression of the BALM morningness-eveningness phenotype with a dominant model meta-analysis showed the strongest association with $\mathrm{rs} 2482705$ ( $\mathrm{p}=8.6 \mathrm{E}-05$,

Table 4. Alleles for rs2482705 (NFIL3) and rs3828057 (RORC) by phenotype

\begin{tabular}{lcccc}
\hline \multirow{2}{*}{ Phenotype } & & rs2482705 & Total \\
\cline { 2 - 4 } & GG allele & AG allele & AA allele & $353(100 \%)$ \\
DSPS case & $277(78.5 \%)$ & $68(19.3 \%)$ & $8(2.3 \%)$ & $326(100 \%)$ \\
Control & $222(68.1 \%)$ & $91(27.9 \%)$ & $13(4 \%)$ & $679(100 \%)$ \\
Total & $499(73.5 \%)$ & $159(23.4 \%)$ & $21(3.1 \%)$ & Total \\
\cline { 2 - 4 } Phenotype & GG allele & rs3828057 & AA allele & $110(100 \%)$ \\
DSPS case & $49(44.5 \%)$ & AG allele & $21(19.1 \%)$ & $627(100 \%)$ \\
Control & $182(29.0 \%)$ & $40(36.4 \%)$ & $128(20.4 \%)$ & $737(100 \%)$ \\
Total & $231(31.3 \%)$ & $357(50.2 \%)$ & $73 \%)$ \\
\hline
\end{tabular}

From the DSPS-case-control sample, the number of participants homozygotic for the common alleles of rs2482705 (NFIL3) and rs3828057 $(R O R C)$ or carrying a minor allele are tabulated versus participants with the DSPS phenotype or their comparable controls. Note that minor (A) alleles were more common among controls than DSPS cases. Participants $(\mathrm{N}=47)$ with equivocal phenotypes were not included

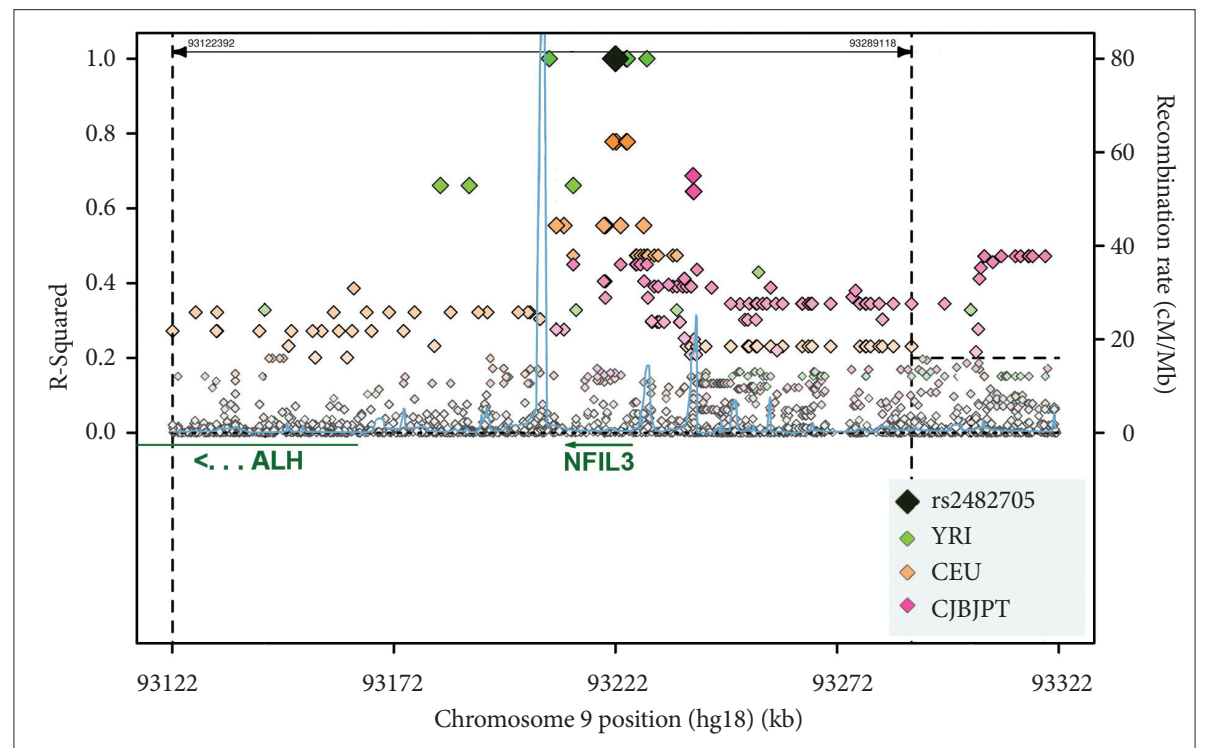

Figure 6. Linkage disequilibrium of NFIL3 by ancestry. Linkage $\left(r^{2}\right)$ of NFIL3 rs2482705 is shown, compiled and edited from SNAP plots ${ }^{48}$ for CEU, CHBJPT, and YRI ancestry groups within the 1000 Genome Pilot 1 data base. Linkage with $r^{2}>0.2$ ranges in the 3 ancestry groups from approximately $100 \mathrm{~K} 5$ ' to rs 2482705 to $100 \mathrm{~K} 3^{\prime}$ downstream to rs 2482705 . There is considerable variation in the linkage patterns in the different ancestry groups, which may be important, considering that the association of rs2482705 with the DSPS phenotype and the BALM in our samples of European ancestry were not supported among non-Europeans. Haploview ${ }^{46}$ lists 6 SNPs (one 5', four intronic, and one 3') with which rs2482705 is strongly linked in a sample of European ancestry (not counting rs33973463). Rs33973463, to which rs2482705 is well-linked (Spearman rho=0.669), is a synonymous coding SNP which may create an ESE splice promotion site. NFIL3 is a competitive inhibitor at D-box transcription factor binding sites. We have not discerned a specific role for rs2482705, an intronic SNP. It is plausible that one or more of the linked SNPs are responsible for the association of rs2482705 with DSPS. 
Bonferroni $\mathrm{p}=0.053$ ), with 2 nominally significant SNPs in NFIL3 by meta-analysis (Figures 8 and 9). Similar results were obtained with the Horne-Östberg scale (see below). Further nominal association with the BALM phenotype was supported by 6 NFIL3 SNPs in the DSPS-case-control sample and 4 SNPs in the Sleep-Center sample (Supplement 1). Metaanalysis for association of the BALM phenotype with SNPs in $R O R C$ was supported only by nominal significance of rs 3828057 ( $\mathrm{p}=0.00189$, Bonferroni NS). Although neither rs 2482705 nor rs3828057 were significantly related to bipolar probability scores in the overall European portion of the DSPScase-control sample $(n=466)$, the minor alleles which appeared protective for DSPS tended to appear somewhat protective for bipolar probability score. Further replication in larger samples would be needed to more accurately assess the strength of these associations.

\section{Strength of genetic associations with DSPS and eveningness}

To assess the strength of association of multiple SNPs combined with the DSPS phenotype and with the BALM morningness-eveningness scale, supplemental multiple linear regressions were computed in SPSS12.0, controlling for age, gender, and ancestry-informative markers, including only participants of European ancestry $<60$ years of age. The measures of association were the partial correlations, followed by the

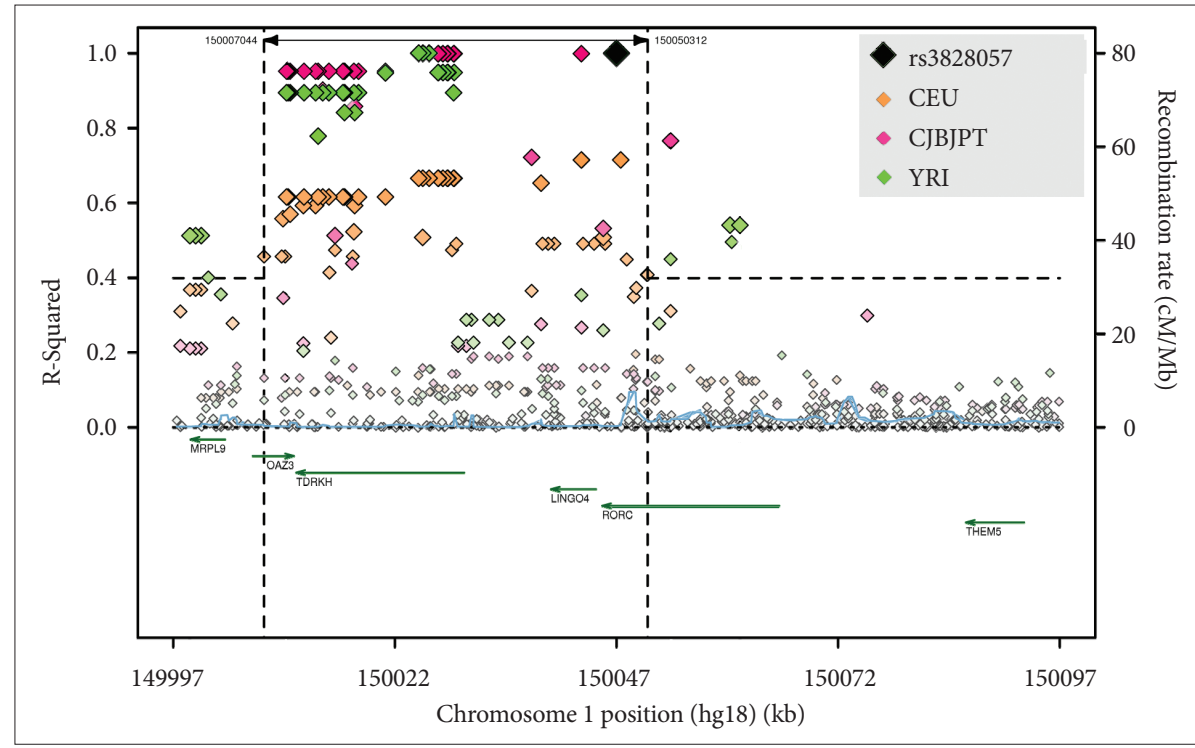

Figure 7. Linkage disequilibrium of $R O R C$ by ancestry. Linkage $\left(\mathrm{r}^{2}\right)$ of $R O R C$ rs 3828057 is shown, compiled and edited from SNAP plots ${ }^{48}$ for CEU, CHBJPT, and YRI ancestry groups within the 1000 Genome Pilot 1 data base. Linkage with $r^{2}>0.4$ ranges in the 3 ancestry groups from the 5' part of RORC to SNPs and genes about 40K downstream to rs3828057. Green arrows show genes in the region with their direction of transcription. RORC binds at RRE transcription factor binding sites on CLOCK, ARNTL, NFIL3, and RORC itself, among others, promoting transcription with complex influences on circadian feedback loops. ${ }^{56}$ The roles of RORC in immunology have attracted the most interest. No specific role of rs3828057 has been discerned, and considering the substantial linkage to several adjacent genes, further investigation will be needed to confirm that RORC actively influences DSPS.

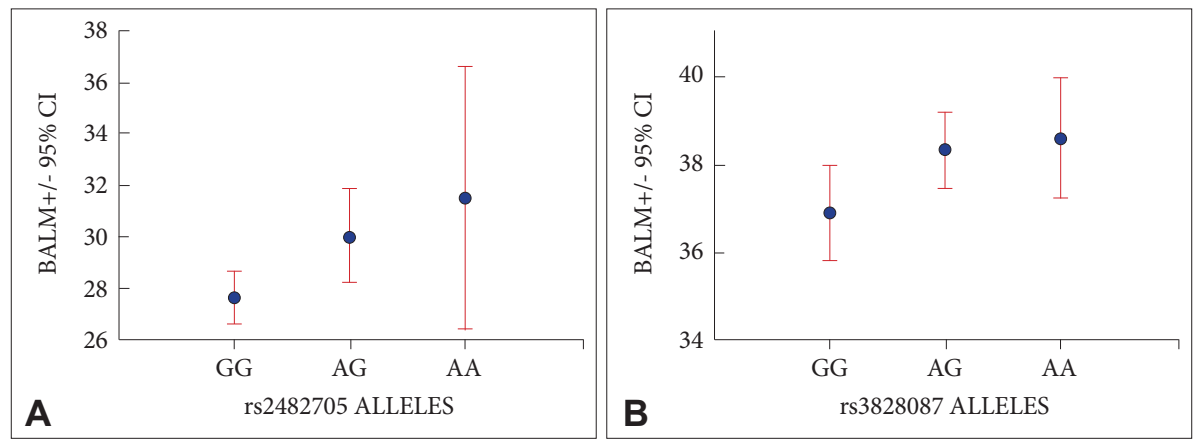

Figure 8. BALM morningness-eveningness scores are shown for DSPS cases and their controls. Alleles of rs2482705 (A) and Alleles of rs3828057 (B). BALM scores <28 indicate marked eveningness, e.g., DSPS. Scores $\geq 28$ indicate the normal range or greater morningness. The means (blue circles) and $95 \%$ confidence intervals (red bars) are shown for homozygotes with the common alleles (left), heterozygotes (center), and for those uncommon homozygotes with the minor alleles (right). For both SNPs, those with 1 or 2 minor alleles (AG or AA) had BALM means outside the confidence limits for the homozygotes (GG) of the common allele, suggesting that minor alleles were associated with dominant protection from DSPS. 
adjusted $\mathrm{R}^{2}\left(\bar{R}^{2}\right)$, to indicate the additional explanatory value the combined SNPs might contribute to the multivariate model. In the DSPS-case-control sample, partial correlations for MTNR1B rs10830962, CRY2 rs11605924, RORA rs12443044, NFIL3 rs2482705, and RORC rs3828057 associations with the DSPS phenotype were $0.124,-0.126,0.139,-0.205$ and -0.115 (NS), respectively. For the associations with the BALM, the partial correlations were $-0.128,0.104,-0.116,0.161$, and 0.095 (NS) respectively. The combined contributions of the significant SNPs in addition to that of covariates retained in the all-combined multiple regression for the DSPS-case-con-

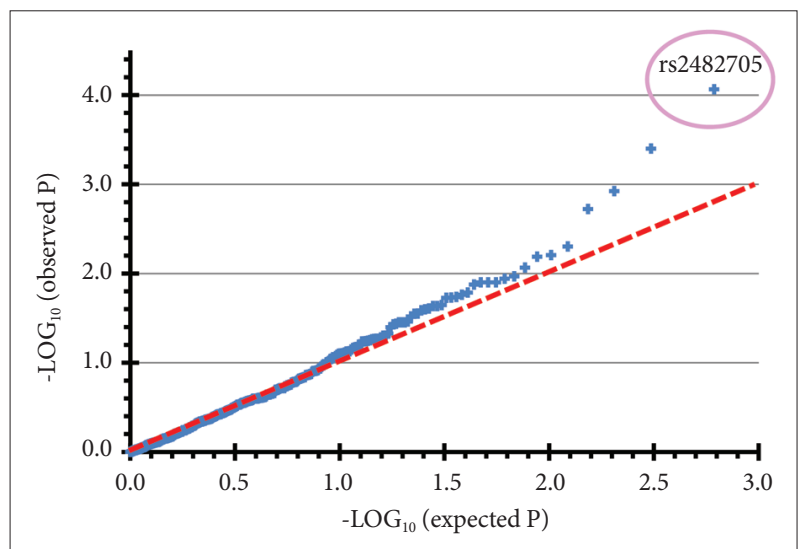

Figure 9. Q-Q plot for the meta-analysis of the BALM phenotype. Quantile-quantile plot of SNP association probabilities for metaanalysis of the BALM phenotype in the combined DSPS-case-control and Sleep Center data sets. See the second worksheet of Supplement 1 for a listing of these $p$ values. The dashed red line shows random expectation for the probability distribution. The pink circle highlights rs2482705. Only 3 other SNPs (rs2292910, rs11605924, and rs3820587 from top to bottom) were notably elevated above the trend line, but apparently almost all the nominally significant SNPs (with observed $-\mathrm{LOG}_{10}[\mathrm{P}]>1.3$ ) were slightly above the trend line, suggesting the possibility of dozens of SNP associations each having small effects. trol sample were adjusted $\bar{R}^{2}=0.093$ for the DSPS phenotype and adjusted $\bar{R}^{2}=0.069$ for BALM. The adjusted $\bar{R}^{2}$ values were slightly less when including participants of age 60 years or more. In the Sleep Center sample, the partial correlations relating DSPS phenotype to these SNPs were $0.129,-0.187$, 0.079 (NS), -0.118 , and -0.178 respectively. The combined adjusted $\bar{R}^{2}$ attributable to the five SNPs was about 0.060 , mainly contributed by rs11605924 and rs3828057. In the Sleep Center sample, the partial correlations relating these SNPs to the BALM were -0.053 (NS), 0.138, -0.088 (NS), 0.150, and 0.121 , with combined adjusted $\bar{R}^{2}$ attributable to the significant SNPs estimated at 0.030 . Unfortunately, the custom Illumina Golden Gate chips did not adequately assay the pattern of associated alleles of BHLHE40, which might have contributed additionally to $\bar{R}^{2}$.

\section{BALM MES compared with Horne-Östberg scale factors}

To explore which phenotyping method was best-associated with a genetic polymorphism influencing delayed sleep, the BALM and Horne-Östberg morningness-eveningness scales, five Horne-Östberg scale subfactors ${ }^{33}$ and the DSPS phenotype were contrasted for their associations with NFIL3 rs2482705, using comparable linear regressions, with dominant models corrected for genomic inflation (Table 5). It appeared that the DSPS case vs. control phenotype was substantially more significantly associated with rs2482705 than the two morningness eveningness scales, which performed about equally well. In some comparisons, the Horne-Östberg scale performed slightly better and in others (not shown), the BALM performed slightly better, so the two scales may be roughly equivalent for studies of genetic association. None of the 5 Horne-Östberg scale factors performed as well as the

Table 5. Genetic association of the Horne-Östberg and BALM scales compared

\begin{tabular}{lccc}
\hline \multicolumn{1}{c}{ Association tested } & Horne-Östberg Items & Nominal P & N participants \\
\hline Horne-Östberg (H-Ö) & All & 0.0015 & 526 \\
BALM & N/A & 0.0018 & 526 \\
H-Ö factor 1 & $9,11,15,17,18,19$ & 0.0033 & 526 \\
H-Ö factor 2 & $4,5,7$ & 0.0069 & 526 \\
H-Ö factor 3 & $2,10,12$ & 0.0058 & 526 \\
H-Ö factor 4 & 3,13 & 0.0095 & 526 \\
H-Ö factor 5 & 1 & 0.0028 & 526 \\
H-Ö-set test & All & 546 \\
BALM-set test & All & 0.0035 & 546 \\
DSPS phenotype & N/A & 0.0160 & $5.23 \mathrm{E}-05$ \\
DSPS phenotype-set test & N/A & 0.0037 & 504 \\
\hline
\end{tabular}

The association of NFIL3 rs2482705 is contrasted for the Horne-Östberg and BALM morningness-eveningness scales, for 5 factors of the Horne-Östberg scale described by Rhee et al, ${ }^{33}$ and for the DSPS phenotype. The strength of the NFIL3 gene-wise PLINK-set tests are likewise contrasted for the two morningness-eveningness scales and the DSPS phenotype 
overall 19-item scale. The BALM scale has only one factor. ${ }^{33}$ The three phenotyping methods were also employed in PLINK genewise-set tests ${ }^{43}$ for NFIL3. The - set tests did not elicit a reliable superiority of one phenotype over another, especially since correction for genomic inflation was not offered in this test.

\section{Objective sleep phase and DSPS}

An objective estimate of actigraphically-inferred sleep acrophase was computed for 190 of the DSPS cases. ${ }^{30}$ No SNP approached Bonferroni significance in this analysis, in which the range of sleep phases was limited because actigraphs were collected from DSPS cases only (no controls). Likewise, no robust genetic association with questionnaire wake-up times was identified in the group of DSPS cases and their controls.

\section{Analysis of OPN4 P10L}

Roecklein and colleagues reported that rs2675703 (the OPN4 P10L polymorphism) interacted with seasonal day length to influence sleep onset, ${ }^{50}$ but in so far as that analysis could be repeated in our DSPS-case-control sample, we found no significant association in additive or recessive models of rs2675703 with the BALM, nor did we find any evidence of association of rs2675703 with DSPS. Only 7 participants with TT recessive genotypes were available for the analysis in interaction with day length. In the subgroup of the DSPS-casecontrol sample whose completed SIGH-SAD questionnaires suggested presence of seasonal affective disorder, no unusual associations of delay phenotypes with polymorphisms were identified. In the Sleep-Center data (for which day length data were not available), the rare TT recessive model was associated with a slightly higher BALM score (that is, greater morningness) with $\mathrm{p}=0.037$, with age, QIDS-SR, and gender controlled, but significance was lost if a strong interaction between gender and genotype was included in the model.

\section{Reported results that were not replicated}

We have emphasized polymorphisms associated with delayed sleep phase which met strict Bonferroni criteria for multiple testing, and which largely replicated in our two participant samples in both DSPS and BALM phenotypes, but we are reminded of the assertion that most reported associations prove false. ${ }^{51}$ Extensive independent replication of results such those reported here is needed. In this context, it is worth noting that our data did not replicate the much-reported (but not always replicated) associations of morningness-eveningness or DSPS with PER3 rs10462020 and a PER3 VNTR linked to rs10462020, rs57875989, ${ }^{52,53}$ or the association with the C3111T CLOCK SNP rs1801260, which is widely-linked throughout CLOCK. ${ }^{25,27}$ We do not consider the nominally significant as- sociation of the BALM with CLOCK C3111T to be a replication, because the direction of association appeared the reverse of the initial report. ${ }^{25}$ To be sure, the older age and comorbidities of our Sleep Center sample may have impeded replication, along with the mixed ancestry of both samples. We failed in our meta-analysis to replicate other reported associations of phase delay with PER2 rs2304672, ${ }^{54}$ or rs7221412 near PER $1,{ }^{28}$ and we did not replicate the reported CLOCK rs 1801260 interaction with GNB3 rs5443. ${ }^{55}$

\section{DISCUSSION}

\section{Role of DSPS loci in circadian oscillation}

In summary, we found several circadian gene loci that promote phase delay, probably including the associated-allele pattern of BHLHE40. The BHLHE40 allele pattern is likewise associated with non-24-hour sleep-wake cycles. BHLHE40 inhibits the positive limb of a core circadian transcriptiontranslation feedback loop by competitively inhibiting ARNTL-CLOCK transcription factor binding to E-box promoter sites. NFIL3 inhibits transcription factor binding at D-boxes, which promote transcription of genes on both positive and negative limbs of the circadian feedback loops, whereas RORC binding at RRE sites may promote the positive limb acting through CLOCK-ARNTL transcription, though SNPs in both NFIL3 and RORC were associated with relative phase advance.

Our findings associating NFIL3 and RORC and a BHLHE40 allele group to DSPS and non-24-hour sleep-wake rhythms suggest polymorphisms in both positive and negative limbs of a primary circadian oscillator feedback loop (Figure 10A). Indeed, since BHLHE40, CRY1, NFIL3, and NR1D1 all themselves have E-boxes in their promoters, ${ }^{56}$ these genes take part in positive and negative limbs of more extensive feedback loops of such complexity that dozens or even hundreds of additional elements may be involved. Each year we learn of new elements in a circadian oscillatory network which is now suspected to involve hundreds of genes, ${ }^{57}$ several interlocking transcriptional feedback loops, phosphorylation by several kinases, microRNA effects, regulation of acetylation and deacetylation of DNA and histones, nuclear and cytoplasmic transfer of RNA and proteins, ubiquitin-related metabolism of proteins, redox states, neurotransmitter effects at cell membranes, calcium channels, intercellular coupling, and other complexities. ${ }^{58}$ The role of specific alleles in non-24-hour rhythms and DSPS could be explored in cell cultures, which display delaying and free-running circadian rhythms related to the donor's phenotype. ${ }^{22,26,59,60}$ For example, over-expression of BHLHE40 produced phase delays in cell cultures. ${ }^{61}$ Once the genetic pathways are clarified, the information may 


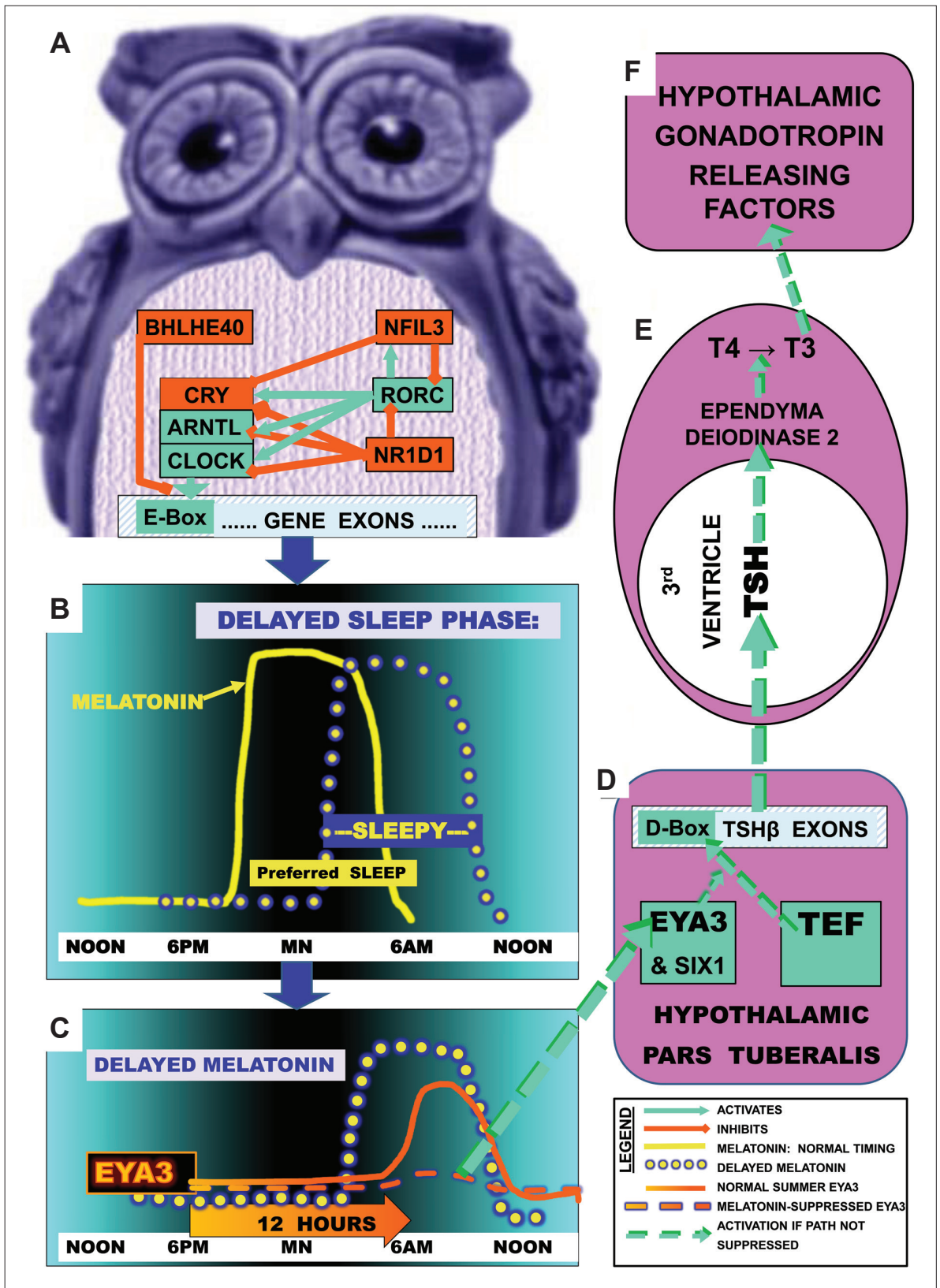

Figure 10. Mechanisms by which DSPS-associated SNPs might lead to photoperiodic disturbances. A: Part of the circadian gene network is shown which promotes transcription through pathways leading to E-box activation (green) or which deactivates transcription and E-box promoter action (red). B: The yellow line illustrates normal melatonin secretion commencing shortly before the preferred nocturnal sleep time and terminating about the time of awakening near dawn, so that preferred sleep times and sleepiness normally correspond. The yellow dotted line illustrates how in DSPS, melatonin secretion may become delayed, consequently delaying sleepiness. C: The gene EYA3 reaches a sharp peak in pars tuberalis transcription about 12 hours after darkness onset (solid orange line), but if melatonin is still elevated (in winter or DSPS), the EYA3 peak is largely suppressed (dashed orange line). ${ }^{81} \mathrm{D}$ : After short nights in summer, EYA3 and SIX1 coactivate TEF at a D-box on the TSH $\beta$ promoter. TSH $\beta$ hybridizes with TSHa, releasing active TSH into 3rd ventricle CSF. ${ }^{81} \mathrm{E}$ : TSH circulates retrograde to promote deiodinase 2 which converts T4 to T3. F: T3 promotes synthesis and release of gonadotropin hormones, implementing summer reproduction and good mood.

be exploited for diagnostic tests and possibly for genetic therapies for DSPS and non-24-hour rhythms.

\section{Circadian phase delay and bipolar disorder}

Some of these same alleles associated with DSPS may be associated with bipolar disorder. ${ }^{62}$ Depression is often the most disabling aspect of bipolar illness. Both unipolar and bipolar depression are associated with eveningness and delayed sleep during depressive episodes. Attention deficit hyperactivity disorder (ADHD), which may be genetically associated with bipolar disorder, may perhaps likewise be associated with phase delays. A pleiotropy between non-24-hour rhythms and bi- 
polar disorder was hypothesized by the late Franz Halberg almost 50 years ago, ${ }^{63}$ with the suggestion that circadian desynchronization or malsynchronization causes the mood disturbances. Indeed, the manic phase of bipolar disorder may be associated with relative phase advance, conceivably produced by transiently delaying non-24-hour components which wrap around the 24 hours to a phase-advanced orientation. A recent study suggested that as with cells from DSPS patients, fibroblasts cultures from bipolar patients tend to display slow (delaying) circadian metabolic cycles in tissue culture. ${ }^{24}$ Another study had a similar finding but too few participants to reach significance. ${ }^{64}$ NR1D1 (a gene protein associated with bipolar disorder ${ }^{65,66}$ inhibits RRE elements in ARNTL-CLOCK, indirectly inhibiting E-box activation. Consistent with these considerations, a CLOCK dominant negative mutation in mice, which may produce phase delay or delaying non-24-hour rhythms ${ }^{67}$ has been claimed to be a possible rodent model for mania. ${ }^{68}$ The ASMT rs $4446909 \mathrm{G}$ allele promotes phase delay possibly by delaying melatonin synthesis, thus promoting depression and bipolar disorder. ${ }^{32,69} \mathrm{An}$ altered circadian relationship of BHLHE40 and PER2 was suggested in assays of depressed brains ${ }^{70}$ and may interact with CLOCK and CRY1 in bipolar disorder. Note also that rs3828057 in $R O R C$ had a nominally significant association with the QIDS-SR depression scale in the DSPS case-control sample, reflecting mainly unipolar depression, and NFIL3 rs7021746 was nominally associated with QIDS-SR by metaanalysis of three samples. ${ }^{34}$ TNF rs 1800629 was nominally linked by meta-analysis to both the DSPS phenotype and to the QIDS-SR, and PPARGC1B rs7732671 showed some nominal linkage to both these phenotypes. ${ }^{34}$ Such findings, though not statistically robust nor significant by Bonferroni criteria, suggest that unipolar depression in common with bipolar disorder might to some extent be linked to polymorphisms causing phase delays.

Evidence is accumulating that delayed melatonin offset is associated with unipolar depressions, ${ }^{7,71-75}$ as suggested in Figure 10. The current results indicate that melatonin offset could become delayed due in part to several polymorphisms which delay circadian rhythm systems. Conversely, it is well known that a person's illumination exposure, particularly exposure to bright morning light, may cause melatonin secretion to terminate earlier in the morning, not only because bright light suppresses melatonin acutely, but also because it advances the "morning oscillator" component of the complex circadian oscillator composed of particular cell groups within the suprachiasmatic nuclei. ${ }^{76,77}$ Morning bright light tends to be antidepressant. ${ }^{78}$ In general, effects of bright light on mood seem to be mediated through retino-hypothalamic tract effects on the suprachiasmatic nucleus, which in turn regulates the timing of melatonin release from the pineal. Likewise, suppression of morning melatonin by propranolol can be antidepressant. ${ }^{79}$

\section{Circadian phase delay and photoperiodism}

In summer-breeding mammals, winter responses related to a late dawn, which prolongs morning melatonin secretion, offer a photoperiodic model of mood disorders. ${ }^{80,81}$ We theorize that phase-delayed melatonin offset could mediate depression partly through these photoperiodic mechanisms, the neuropharmacology of which is now better understood (Figure 10). The hypothalamic pars tuberalis has a particularly rich supply of melatonin receptors, which may be the primary target of melatonin effects on seasonal photoperiodic responses. In the short nights of summer, melatonin secretion is terminated by an early dawn. As illustrated in Figure 10C, about 12 hours after sunset, EYA3 rises to a peak in the pars tuberalis. EYA3 with SIX1 coactivate TEF promoter effects at a D-box of the TSH $\beta$ subunit. ${ }^{80,82}$ Combining with TSH $\alpha$, active TSH circulates retrograde in the third ventricle, entering tanycytes in the ependymal lining and activating transcription of deiodinase 2, which then converts T4 to T3. T3 activates hypothalamic secretion of gonadotropins, stimulating summer breeding. If termination of morning melatonin secretion is delayed, either by the later dawn of winter or perhaps by genetically-mediated circadian phase delays (Figure 10C), melatonin suppresses the peak of EYA3 and thus reduces TSH transcription in pars tuberalis, leading to reduced hypothalamic synthesis of T3, and consequent inhibition of gonadotropin releasing factors. ${ }^{80-84} \mathrm{~T} 3$ may directly influence hypothalamic cells secreting gonadotropin releasing and inhibiting factors as well as kisspeptin. ${ }^{85}$ However, there remain many unresolved issues in the molecular biology of photoperiodism, such as how the circadian system, perhaps through melatonin onset, synchronizes the peak time of EYA3 release.

Seasonal mechanisms uncovered in laboratory animals may not be exact models for human winter depressions nor for the broader problems of unipolar and bipolar depression which are not always seasonal. Additional circadian mechanisms which are not photoperiodic pathways might regulate biologic components of depression such as reduced activity of the dopamine system and hypothalamic-pituitary-adrenal over-activity (hypercortisolism). Transcription of $M A O A$, a crucial enzyme metabolizing dopamine, is regulated by CLOCKARNTL transcription factor binding on MAOA promoter Eboxes ${ }^{86}$ and thus, by the positive limb of the circadian oscillator feedback loop. How the genetic polymorphisms we have observed or photoperiod might affect MAOA is not yet established. ENCODE data list a weak BHLHE40 binding site in the promoter of NR3C1, a crucial glucocorticoid receptor related to hypercortisolism. Likewise, hypercortisolism is 
regulated by very strong circadian modulation through a SCN-paraventricular pathway, presumably mediating how morning light elevates cortisol. ${ }^{87,88}$ In contrast, both cryptochrome genes inhibit glucocorticoid receptor activation. ${ }^{89}$

In the future, genetic testing might clarify when circadian polymorphisms contribute to sleep disturbances, depression, and bipolar disorder, thus inspiring improved interventions to treat these disorders. Meanwhile, there are behavioral interventions and applications of bright light and melatonin that may be useful in palliating both non-24-hour circadian tendencies and DSPS. ${ }^{90}$ Bright light is already being used successfully for depression and bipolar disorder. ${ }^{78}$ Eventually, genetic understanding may guide improved pharmacologic, lighting, behavioral and genetic interventions to help those affected become more healthy, wealthy, and wise.

\section{Supplementary Materials}

The online-only Data Supplement is available with this article at http://dx.doi.org/10.4306/pi.2014.11.4.345

\section{Acknowledgments}

Supported by U.S. National Institutes of Health grants HL071123, 5 UL1 RR025774, AG030474, MH078151, MH08180, and MH59567 and by Scripps Clinic Academic funds. Alexandra P. Grizas and Elizabeth K. Hahn, RN contributed to Sleep Center participant recruitment and human subjects protection, questionnaire and DNA sample collections, and data base development. Arthur Dawson, M.D. and John W. Cronin, M.D. recruited and diagnosed Sleep Center patients and provided scientific consultation during the research. The authors declare that they have no competing financial interests.

\section{REFERENCES}

1. Gale C, Martyn C. Larks and owls and health, wealth, and wisdom. BMJ 1998;317:1675-1677.

2. Roberts RD, Kyllonen PC. Morningness-eveningness and intelligence: early to bed, early to rise will likely make you anything but wise! Pers Individ Dif 1999;27:1123-1133.

3. Merikanto I, Lahti T, Kronholm E, Peltonen M, Laatikainen T, Vartiainen $\mathrm{E}$, et al. Evening types are prone to depression. Chronobiol Int 2013;30:719-725.

4. Lee HJ, Rex KM, Nievergelt CM, Kelsoe JR, Kripke DF. Delayed sleep phase syndrome is related to seasonal affective disorder. J Affect Disord 2011;133:573-579.

5. Giglio LM, Magalhaes PV, Andersen ML, Walz JC, Jakobson L, Kapczinski F. Circadian preference in bipolar disorder. Sleep Breath 2010;14: 153-155.

6. Robillard R, Naismith SL, Rogers NL, Ip TK, Hermens DF, Scott EM, et al. Delayed sleep phase in young people with unipolar or bipolar affective disorders. J Affect Disord 2013;145:260-263.

7. Lewy AJ. Circadian misalignment in mood disturbances. Curr Psychiatry Rep 2009;11:459-465.

8. Murray CJ, Vos T, Lozano R, Naghavi M, Flaxman AD, Michaud C, et al. Disability-adjusted life years (DALYs) for 291 diseases and injuries in 21 regions, 1990-2010: a systematic analysis for the Global Burden of Disease Study 2010. Lancet 2012;380:2197-2223.

9. Saxvig IW, Pallesen S, Wilhelmsen-Langeland A, Molde H, Bjorvatn B. Prevalence and correlates of delayed sleep phase in high school students. Sleep Med 2012;13:193-199.
10. Randler C. Association between morningness-eveningness and mental and physical health in adolescents. Psychol Health Med 2011;16:29-38.

11. Tzischinsky O, Shochat T. Eveningness, sleep patterns, daytime functioning, and quality of life in Israeli adolescents. Chronobiol Int 2011; 28: $338-343$.

12. O'Brien EM, Mindell JA. Sleep and risk-taking behavior in adolescents. Behav Sleep Med 2005;3:113-133.

13. Reid KJ, Jaksa AA, Eisengart JB, Baron KG, Lu B, Kane P, et al. Systematic evaluation of Axis-I DSM diagnoses in delayed sleep phase disorder and evening-type circadian preference. Sleep Med 2012;13:1171-1177.

14. Winkelman J, Kotagal S, Olson E, Scammel T, Schenck C, Spielman A. The International Classification of Sleep Disorders, Second Edition, Pocket Version: Diagnostic and Coding Manual. Westchester, IL: American Academy of Sleep Disorders; 2006.

15. Kripke DF, Langer RD, Elliott JA, Klauber MR, Rex KM. Mortality related to actigraphic long and short sleep. Sleep Med 2011;12:28-33.

16. Brown FM. Psychometric equivalence of an improved Basic Language Morningness (BALM) scale using industrial population within comparisons. Ergonomics 1993;36:191-197.

17. Koskenvuo M, Hublin C, Partinen M, Heikkila K, Kaprio J. Heritability of diurnal type: a nationwide study of 8753 adult twin pairs. J Sleep Res 2007;16:156-162.

18. Barclay NL, Eley TC, Buysse DJ, Archer SN, Gregory AM. Diurnal preference and sleep quality: same genes? A study of young adult twins. Chronobiol Int 2010;27:278-296.

19. Vink JM, Groot AS, Kerkhof GA, Boomsma DI. Genetic analysis of morningness and eveningness. Chronobiol Int 2001;18:809-822.

20. Randler C. Morningness-eveningness comparison in adolescents from different countries around the world. Chronobiol Int 2008;25:1017-1028.

21. Barclay NL, Eley TC, Parsons MJ, Willis TA, Gregory AM. Monozygotic twin differences in non-shared environmental factors associated with chronotype. J Biol Rhythms 2013;28:51-61.

22. Brown SA, Kunz D, Dumas A, Westermark PO, Vanselow K, TilmannWahnschaffe A, et al. Molecular insights into human daily behavior. Proc Natl Acad Sci U S A 2008;105:1602-1607.

23. Micic G, de Bruyn A, Lovato N, Wright H, Gradisar M, Ferguson S, et al. The endogenous circadian temperature period length (tau) in delayed sleep phase disorder compared to good sleepers. J Sleep Res 2013;22: 617-624.

24. McCarthy MJ, Wei H, Marnoy Z, Darvish RM, McPhie DL, Cohen BM, et al. Genetic and clinical factors predict lithium's effects on PER2 gene expression rhythms in cells from bipolar disorder patients. Transl Psychiatry 2013;3:e318.

25. Katzenberg D, Young T, Finn L, Lin L, King DP, Takahashi JS, et al. A clock polymorphism associated with human diurnal preference. Sleep 1998;21:569-576.

26. Ebisawa T. Analysis of the molecular pathophysiology of sleep disorders relevant to a disturbed biological clock. Mol Genet Genomics 2013; 288:185-193.

27. Cade BE. Variation and Selection in Human Circadian Clock Genes. Dissertation, University of Surrey; 2010.

28. Lim AS, Chang AM, Shulman JM, Raj T, Chibnik LB, Cain SW, et al. A common polymorphism near PER1 and the timing of human behavioral rhythms. Ann Neurol 2012;72:324-334.

29. Jones CR, Huang AL, Ptacek LJ, Fu YH. Genetic basis of human circadian rhythm disorders. Exp Neurol 2013;243:28-33.

30. Kripke DF, Rex KM, Ancoli-Israel S, Nievergelt CM, Klimecki W, Kelsoe JR. Delayed sleep phase cases and controls. J Circadian Rhythms 2008;6:6.

31. Kripke DF, Shadan FF, Dawson A, Cronin JW, Jamil SM, Grizas AP, et al. Genotyping sleep disorders patients. Psychiatry Investig 2010;7:36-42.

32. Kripke DF, Nievergelt CM, Tranah GJ, Murray SS, McCarthy MJ, Rex $\mathrm{KM}$, et al. Polymorphisms in melatonin synthesis pathways: possible influences on depression. J Circadian Rhythms 2011;9:8.

33. Rhee MK, Lee HJ, Rex KM, Kripke DF. Evaluation of two circadian 
rhythm questionnaires for screening for the delayed sleep phase disorder. Psychiatry Investig 2012;9:236-244.

34. Kripke DF, Nievergelt CM, Tranah GJ, Murray SS, Rex KM, Grizas AP, et al. FMR1, circadian genes and depression: suggestive associations or false discovery? J Circadian Rhythms 2013;11:3.

35. Horne JA, Östberg O. A self-assessment questionnaire to determine morningness-eveningness in human circadian rhythms. Int J Chronobiol 1976;4:97-110.

36. Terman M, Rifkin JB, Jacobs J, White TM. Morningness-Eveningness Questionnaire (Revised). New York: New York State Psychiatric Institute; 2008

37. Hirschfeld RM, Holzer C, Calabrese JR, Weissman M, Reed M, Davies M, et al. Validity of the Mood Disorder Questionnaire: a general population study. Am J Psychiatry 2003;160:178-180.

38. Rush AJ, Trivedi MH, Ibrahim HM, Carmody TJ, Arnow B, Klein DN, et al. The 16-item Quick Inventory of Depressive Symptomatology (QIDS), clinical rating (QIDS-C), and self-report (QIDS-SR): a psychometric evaluation in patients with chronic major depression. Biol Psychiatry 2003;54:573-583.

39. Ando K, Kripke DF, Ancoli-Israel S. Delayed and advanced sleep phase symptoms. Isr J Psychiatry Relat Sci 2002;39:11-18.

40. Kripke DF, Klimecki W. EF015893-EF015905 and EF028070. GenBank Nucleotide. 9-21-2006. http://www.ncbi.nlm.nih.gov/nuccore/ef015893 and following, NCBI.

41. Gottlieb DJ, O'Connor GT, Wilk JB. Genome-wide association of sleep and circadian phenotypes. BMC Med Genet 2007;8(Suppl 1):S9.

42. Nievergelt CM, Maihofer AX, Shekhtman T, Libiger O, Wang X, Kidd $\mathrm{KK}$, et al. Inference of human continental origin and admixture proportions using a highly discriminative ancestry informative 41-SNP panel. Investig Genet 2013;4:13.

43. Purcell S, Neale B, Todd-Brown K, Thomas L, Ferreira MA, Bender D, et al. PLINK: a tool set for whole-genome association and populationbased linkage analyses. Am J Hum Genet 2007;81:559-575.

44. Storey JD, Tibshirani R. Statistical significance for genomewide studies. Proc Natl Acad Sci U S A 2003;100:9440-9445.

45. Honma S, Kawamoto T, Takagi Y, Fujimoto K, Sato F, Noshiro M, et al. Dec1 and Dec2 are regulators of the mammalian molecular clock. Nature 2002;419:841-844.

46. Barrett JC, Fry B, Maller J, Daly MJ. Haploview: analysis and visualization of LD and haplotype maps. Bioinformatics 2005;21:263-265.

47. Kripke DF, Mullaney DJ, Atkinson M, Wolf S. Circadian rhythm disorders in manic-depressives. Biol Psychiatry 1978;13:335-351.

48. Johnson AD, Handsaker RE, Pulit S, Nizzari MM, O'donnell CJ, de Bakker PIW. SNAP: a web-based tool for identification and annotation of proxy SNPs using HapMap. Bioinformatics 2008;24:2938-2939.

49. Torkamani A. Scripps Genome Adviser: Annotation and Distributed Variant Interpretation Server. 2013. http://genomics.scripps.edu/adviser/, Scripps Translational Science Institute. Accessed July 25, 2013.

50. Roecklein KA, Wong PM, Franzen PL, Hasler BP, Wood-Vasey WM, Nimgaonkar VL, et al. Melanopsin gene variations interact with season to predict sleep onset and chronotype. Chronobiol Int 2012;29: 1036-1047.

51. Ioannidis JP. Why most published research findings are false. PLoS Med 2005;2:e124.

52. Ebisawa T, Uchiyama M, Kajimura N, Mishima K, Kamei Y, Katoh M, et al. Association of structural polymorphisms in the human period3 gene with delayed sleep phase syndrome. EMBO Rep 2001;2:342-346.

53. Archer SN, Robilliard DL, Skene DJ, Smits M, Williams A, Arendt J, et al. A length polymorphism in the circadian clock gene Per3 is linked to delayed sleep phase syndrome and extreme diurnal preference. Sleep 2003;26:413-415.

54. Carpen JD, Archer SN, Skene DJ, Smits M, von Schantz M. A single-nucleotide polymorphism in the 5'-untranslated region of the hPER2 gene is associated with diurnal preference. J Sleep Res 2005;14:293-297.

55. Lee HJ, Paik JW, Kang SG, Lim SW, Kim L. Allelic variants interaction of CLOCK gene and G-protein beta3 subunit gene with diurnal preference. Chronobiol Int 2007;24:589-597.

56. Minami Y, Ode KL, Ueda HR. Mammalian circadian clock: the roles of transcriptional repression and delay. Handb Exp Pharmacol 2013;217: 359-377.

57. Zhang EE, Liu AC, Hirota T, Miraglia LJ, Welch G, Pongsawakul PY, et al. A genome-wide RNAi screen for modifiers of the circadian clock in human cells. Cell 2009;139:199-210.

58. Albrecht U. Timing to perfection: the biology of central and peripheral circadian clocks. Neuron 2012;74:246-260.

59. Pagani L, Semenova EA, Moriggi E, Revell VL, Hack LM, Lockley SW, et al. The physiological period length of the human circadian clock in vivo is directly proportional to period in human fibroblasts. PLoS ONE 2010;5:e13376.

60. Ebisawa T, Numazawa K, Shimada H, Izutsu H, Sasaki T, Kato N, et al. Self-sustained circadian rhythm in cultured human mononuclear cells isolated from peripheral blood. Neurosci Res 2009;66:223-227.

61. Nakashima A, Kawamoto T, Honda KK, Ueshima T, Noshiro M, Iwata $\mathrm{T}$, et al. DEC1 modulates the circadian phase of clock gene expression. Mol Cell Biol 2008;28:4080-4092.

62. Shi J, Wittke-Thompson JK, Badner JA, Hattori E, Potash JB, Willour $\mathrm{VL}$, et al. Clock genes may influence bipolar disorder susceptibility and dysfunctional circadian rhythm. Am J Med Genet B Neuropsychiatr Genet 2008;147B:1047-1055.

63. Halberg F. Physiologic Considerations Underlying Rhythmometry, with Special Reference to Emotional Illness. Symposium on Biological Cycles and Psychiatry. Symposium Bell-Air III. Geneva: Mason et Cie, 1967, p.73-126.

64. Bamne MN, Ponder CA, Wood JA, Mansour H, Frank E, Kupfer DJ, et al. Application of an ex vivo cellular model of circadian variation for bipolar disorder research: a proof of concept study. Bipolar Disord 2013; 15:694-700.

65. Kripke DF, Nievergelt CM, Joo EJ, Shekhtman T, Kelsoe JR. Circadian polymorphisms associated with affective disorders. J Circadian Rhythms 2009;7:2.

66. McCarthy MJ, Nievergelt CM, Shekhtman T, Kripke DF, Welsh DK, Kelsoe JR. Functional genetic variation in the Rev-Erbalpha pathway and lithium response in the treatment of bipolar disorder. Genes Brain Behav 2011;10:852-861.

67. Vitaterna MH, King DP, Chang AM, Kornhauser JM, Lowrey PL, McDonald JD, et al. Mutagenesis and mapping of a mouse gene, Clock, essential for circadian behavior. Science 1994;264:719-725.

68. Roybal K, Theobold D, Graham A, Dinieri JA, Russo SJ, Krishnan V, et al. Mania-like behavior induced by disruption of CLOCK. Proc Natl Acad Sci U S A 2007;104:6406-6411.

69. Etain B, Dumaine A, Bellivier F, Pagan C, Francelle L, Goubran-Botros $\mathrm{H}$, et al. Genetic and functional abnormalities of the melatonin biosynthesis pathway in patients with bipolar disorder. Hum Mol Genet 2012;21:4030-4037.

70. Li JZ, Bunney BG, Meng F, Hagenauer MH, Walsh DM, Vawter MP, et al. Circadian patterns of gene expression in the human brain and disruption in major depressive disorder. Proc Natl Acad Sci U S A 2013; 110:9950-9955.

71. Sekula LK, Lucke JF, Heist K, Czambel K, Rubin RT. Neuroendocrine aspects of primary endogenous depression XV: mathematical modeling of nocturnal melatonin secretion in major depressives and normal controls. Psychiatry Res 1997;69:143-153.

72. Nurnberger JI Jr, Adkins S, Lahiri DK, Mayeda A, Hu K, Lewy A, et al. Melatonin suppression by light in euthymic bipolar and unipolar patients. Arch Gen Psychiatry 2000;57:572-579.

73. Tuunainen A, Kripke DF, Elliott JA, Assmus JD, Rex KM, Klauber MR, et al. Depression and endogenous melatonin in postmenopausal women. J Affect Disord 2002;69:149-158.

74. Emens J, Lewy A, Kinzie JM, Arntz D, Rough J. Circadian misalignment in major depressive disorder. Psychiatry Res 2009;168:259-261. 
75. Meliska CJ, Martinez LF, Lopez AM, Sorenson DL, Nowakowski S, Parry BL. Relationship of morningness-eveningness questionnaire score to melatonin and sleep timing, body mass index and atypical depressive symptoms in peri- and post-menopausal women. Psychiatry Res 2011; 188:88-95.

76. Naito E, Watanabe T, Tei H, Yoshimura T, Ebihara S. Reorganization of the suprachiasmatic nucleus coding for day length. J Biol Rhythms 2008;23:140-149.

77. Honma S, Ono D, Suzuki Y, Inagaki N, Yoshikawa T, Nakamura W, et al. Suprachiasmatic nucleus: cellular clocks and networks. Prog Brain Res 2012;199:129-141.

78. Wirz-Justice A, Benedetti F, Terman M. Chronotherapeutics for Affective Disorders: A Clinician's Manual for Light and Wake Therapy. 2nd Edition. Basel, Switzerland: Karger; 2013.

79. Schlager DS. Early-morning administration of short-acting beta blockers for treatment of winter depression. Am J Psychiatry 1994;151: 1383-1385.

80. Dardente H, Wyse CA, Birnie MJ, Dupre SM, Loudon AS, Lincoln GA, et al. A molecular switch for photoperiod responsiveness in mammals. Curr Biol 2010;20:2193-2198.

81. Hazlerigg D. The evolutionary physiology of photoperiodism in vertebrates. Prog Brain Res 2012;199:413-422.

82. Masumoto KH, Ukai-Tadenuma M, Kasukawa T, Nagano M, Uno $\mathrm{KD}$, Tsujino K, et al. Acute induction of Eya3 by late-night light stimulation triggers TSH $\beta$ expression in photoperiodism. Curr Biol 2010; 20:2199-2206.

83. Prendergast BJ, Pyter LM, Kampf-Lassin A, Patel PN, Stevenson TJ.
Rapid induction of hypothalamic iodothyronine deiodinase expression by photoperiod and melatonin in juvenile Siberian hamsters ( $\mathrm{Ph}$ odopus sungorus). Endocrinology 2013;154:831-841.

84. Stevenson TJ, Prendergast BJ. Reversible DNA methylation regulates seasonal photoperiodic time measurement. Proc Natl Acad Sci U S A 2013;110:16651-16656.

85. Henson JR, Carter SN, Freeman DA. Exogenous $\mathrm{T}_{3}$ elicits long daylike alterations in testis size and the RFamides Kisspeptin and gonadotropin-inhibitory hormone in short-day Siberian hamsters. J Biol Rhythms 2013;28:193-200.

86. Hampp G, Ripperger JA, Houben T, Schmutz I, Blex C, Perreau-Lenz $\mathrm{S}$, et al. Regulation of monoamine oxidase A by circadian-clock components implies clock influence on mood. Curr Biol 2008;18:678-683.

87. Leproult R, Colecchia EF, L'Hermite-Baleriaux M, Van Cauter E. Transition from dim to bright light in the morning induces an immediate elevation of cortisol levels. J Clin Endocrinol Metab 2001;86:151-157.

88. Scheer FAJL, Buijs RM. Light affects morning salivary cortisol in humans. J Clin Endocrinol Metab 1999;84:3395-3398.

89. Lamia KA, Papp SJ, Yu RT, Barish GD, Uhlenhaut NH, Jonker JW, et al. Cryptochromes mediate rhythmic repression of the glucocorticoid receptor. Nature 2011;480:552-556.

90. Wilhelmsen-Langeland A, Saxvig IW, Pallesen S, Nordhus IH, Vedaa O, Lundervold AJ, et al. A randomized controlled trial with bright light and melatonin for the treatment of delayed sleep phase disorder: effects on subjective and objective sleepiness and cognitive function. J Biol Rhythms 2013;28:306-321. 